\title{
Zonal Organization of the Climbing Fiber Projection to the Flocculus and Nodulus of the Rabbit: A Combined Axonal Tracing and Acetylcholinesterase Histochemical Study
}

\author{
J. TAN, N.M. GERRITS, R. NANHOE, J.I. SIMPSON, AND J. VOOGD \\ Department of Anatomy, Erasmus University Rotterdam, 3000 DR Rotterdam, \\ The Netherlands; (J.T., N.M.G., J.V.), General Hospital Middelheim, Department of \\ Neurology, Middelheim, Belgium (R.N.), and Department of Physiology and Biophysics, \\ New York University Medical Center, New York, New York 10016 (J.I.S.)
}

\begin{abstract}
The localization and termination of olivocerebellar fibers in the flocculus and nodulus of the rabbit were studied with anterograde axonal transport methods [wheatgerm agglutininhorseradish peroxidase (WGA-HRP) and tritiated leucine] and correlated with the compartments in the white matter of these lobules delineated with acetylcholinesterase histochemistry (Tan et al. J. Comp. Neurol., 1995, this issue). Olivocerebellar fibers originating from the caudal dorsal cap travel through floccular compartments $\mathrm{FC}_{2}$ and $\mathrm{FC}_{4}$ to terminate as climbing fibers in floccular zones $\mathrm{FZ}_{\mathrm{II}}$ and $\mathrm{FZ}_{\mathrm{IV}}$. Fibers from the rostral dorsal cap and the ventrolateral outgrowth traverse compartments $\mathrm{FC}_{1}$ and $\mathrm{FC}_{3}$, which are interleaved with compartments $\mathrm{FC}_{2}$ and $\mathrm{FC}_{4}$, and terminate in zones $\mathrm{FZ}_{1}$ and $\mathrm{FZ}_{\mathrm{III}}$. Fibers from the rostral pole of the medial accessory olive traverse the $\mathrm{C}_{2}$ compartment and terminate in the $\mathrm{C}_{2}$ zone. $\mathrm{FZ}_{\mathrm{I}-\mathrm{III}}$ extend into the adjoining folium (folium $\mathbf{p}$ ) of the ventral paraflocculus. The $\mathrm{C}_{2}$ zone continues across folium $p$ into other folia of the ventral paraflocculus and into the dorsal paraflocculus. Four compartments and five zones were distinguished in the nodulus. Medial compartment $\mathrm{XC}_{1}$ contains olivocerebellar fibers from the caudal dorsal cap and subnucleus $\beta$ that terminate in the $\mathrm{XZ}_{\mathrm{I}}$ zone. Olivocerebellar fibers from the rostral dorsal cap and the ventrolateral outgrowth occupy $\mathrm{XC}_{2}$ and terminate in $\mathrm{XZ}_{\mathrm{II}}$. The $\mathrm{XC}_{4}$ compartment contains fibers from both the caudal dorsal cap and from the rostral dorsal cap and the ventrolateral outgrowth. The latter terminate in a central portion of the $\mathrm{XZ}_{\mathrm{IV}}$ zone. The dorsomedial cell column projects to the $\mathrm{XZ}_{\mathrm{III}}$ zone, which is present only in the dorsal part of the nodulus. The rostral medial accessory olive projects to the $\mathrm{XZY}$ zone, which occupies the lateral border of the nodulus. These results confirm and extend the conclusions of Katayama and Nisimaru ([1988] Neurosci. Res. $5: 424-438$ ) on the zonal pattern in the olivo-nodular projection in the rabbit. Additional observations were made on the presence of a lateral A zone (Buisseret-Delmas [1988] Neurosci. Res. 5:475-493) in the hemisphere of lobules VI and VII. Retrograde labeling of the nucleo-olivary tract of Legendre and Courville ([1987] Neuroscience 21:877-891) was observed after WGA-HRP injections into the inferior olive including the rostral dorsal cap and the ventrolateral outgrowth. The anatomical and functional implications of these observations are discussed. 1995 Wilev-Liss, Inc.
\end{abstract}

Indexing terms: cerebellum, inferior olive, nucleo-olivary tract, dorsal cap, ventrolateral outgrowth

The flocculus and nodulus are involved in several motor systems relayed by the vestibular nuclei. Best known is the role of the flocculus in the adaptive control of eye movements (Ito, 1972, 1982, 1984; Robinson, 1976; Ito et al., $1982 \mathrm{a}, \mathrm{b} ;$ Nagao, 1983, 1988). The flocculus receives a visual
Accepted Scptember 9, 1994.

Address reprint requests to J. Voogd, Department of Anatomy, Erasmus University Rotterdam, P.O. Box 1738, 3000 DR Rotterdam, The Netherlands. 
input via climbing fibers from the inferior olive. The nodulus receives climbing fibers from both visually and vestibularly dominated subnuclei of the inferior olive. The main output of the flocculus and the nodulus is directed through their Purkinje cell axons to the vestibular nuclei.

Climbing fibers terminating in the flocculus originate from the dorsal cap (dc) and adjoining ventrolateral outgrowth (vlo) of the inferior olive with only minor species differences (Maekawa and Simpson, 1972, 1973; Alley et al., 1975; Maekawa and Takeda, 1976, 1977; Groenewegen and Voogd, 1977; Hoddevik and Brodal, 1977; Kawamura and Hashikawa, 1979; Walberg et al., 1979; Yamamoto, 1979a; Brodal and Brodal, 1982; Gerrits and Voogd, 1982, 1989; Blanks et al., 1983; Sato et al., 1983; Whitworth et al., 1983; Balaban, 1984; Takeda and Maekawa 1984a, 1989a,b; Langer et al., 1985; Bernard, 1987; Blanks, 1990; Ruigrok et al., 1992). They also originate from the rostral pole of the medial accessory olive (MAO; Brodal, 1940; Alley et al., 1975; Hoddevik and Brodal, 1977; Walberg et al., 1979; Yamamoto, 1979a; Brodal and Brodal, 1982; Gerrits and Voogd, 1982; Blanks et al., 1983; Langer et al., 1985; Ruigrok et al., 1992). Climbing fibers to the nodulus and the adjoining part of the uvula take their origin from the dc and vlo, the subnucleus $\beta$, the dorsomedial cell column (dmec), rostral and caudal parts of the MAO, and the ventral leaf of the principal olivary nucleus (Brodal, 1940, 1976; Alley et al., 1975; Groenewegen and Voogd, 1977; Groenewegen et al., 1979; Kawamura and Hashikawa, 1979; Brodal and Kawamura, 1980; Voogd and Bigaré,
1980; Brodal and Brodal, 1981, 1982; Furber and Watson, 1983; Whitworth et al., 1983; Eisenman, 1984; Sato and Barmack, 1985; Bernard, 1987; Walberg et al., 1987; Balaban and Henry, 1988; Katayama and Nisimaru, 1988; Kanda et al., 1989; Kano et al., 1990). Some of the neurons of the dc and the vlo distribute their axons to both the flocculus and the nodulus and uvula (Takeda and Maekawa, 1984a,b, 1989a,b; Maekawa et al., 1989).

Visual signals are sent to the dc/vlo via the nucleus of the optic tract $\left(\mathrm{NO}^{\prime} \mathrm{T}\right)$, the nuclei of the accessory optic system (AOS), and the visual tegmental relay zone (VTRZ). The projection of the optic nerve to the NOT and the nuclei of the AOS is mainly crossed. The caudal part of the dc receives afferent projections from the dorsal (DTN) and interstitial terminal nuclei (ITN; i.e., the interstitial nucleus of the superior fascicle of the AOS; Giolli et al., 1984, 1985, 1988) and the NOT (Mizuno et al., 1973, 1974; Takeda and Maekawa, 1976; Holstege and Collewijn, 1982). This projection is mainly uncrossed; in the rabbit it also involves the subnucleus $\beta$ (Holstege and Collewijn, 1982). The rostral part of the $d c$ and the vlo receive afferent projections from the medial terminal nucleus of the accessory optic system (MTN) and the VTRZ (Maekawa and Takeda, 1977, 1979; Giolli et al., 1984, 1985; Simpson et al., 1988). The major olivary projection from these nuclei is ipsilateral and is derived from the VTRZ. Physiologically the dc/vlo complex can also be subdivided into caudal and rostral parts on the basis of laterality of retinal signals (Maekawa and 'Takeda, 1976, 1977; Ito et al., 1978; Takeda and Maekawa, 1980),

Abbreviations

\begin{tabular}{ll} 
A & cortical zone A \\
Alat & lateral A zone of Buisseret-Delmas (1988) \\
ANS & ansiform lobule \\
ANT & anterior lobule \\
B & cortical zone B \\
$\beta$ & subnucleus beta \\
bc & brachium conjunctivum \\
bp & brachium pontis \\
C & C $_{2}$ zone or compartment \\
cdc & caudal dorsal cap \\
CGM & medial geniculate body \\
CO & cochlear nucleus \\
cp & posterior commissure \\
DAO & dorsal accessory olive \\
DC, dc & dorsal cap \\
dl & dorsal lamella of the principal olive \\
DLP & dorsolateral protuberance \\
DMCC, dmcc & dorsomedial cell column \\
DTN & dorsal terminal nucleus \\
DV & descending vestibular nucleus \\
F & fastigial nucleus \\
FC & floccular compartments 1-4 \\
fII & primary fissure \\
FLO & flocculus \\
fm, m & folium m \\
fp, & folium p \\
FZ & floccular zones I-IV \\
f1 & folium 1 \\
f2 & folium 2 \\
f3 & folium 3 \\
f4 & folium 4 \\
f5 & folium 5 \\
IA & anterior interposed nucleus \\
INT & interstitial nucleus of Cajal \\
IP & posterior interposed nucleus \\
ITN & interstitial terminal nucleus \\
l & lateral \\
I VII & lobule VII \\
I IX & lobule IX, uvula \\
IX & lobule X, nodulus \\
L & lateral cerebellar nucleus \\
& \\
\hline
\end{tabular}

\begin{tabular}{|c|c|}
\hline Lpe & parvicellular part of the lateral cerebellar nucleus \\
\hline LI'N & Jateral terminal nucleus \\
\hline LV & lateral vestibular nucleus \\
\hline $\begin{array}{l}\text { MAO } \\
\text { ML }\end{array}$ & $\begin{array}{l}\text { medial accessory olive } \\
\text { medial lemniscus }\end{array}$ \\
\hline MLF, mlf & $\begin{array}{l}\text { medial lemniscus } \\
\text { medial longitudinal fascicle }\end{array}$ \\
\hline MTN & medial terminal nucleus \\
\hline MV & medial vestibular nucleus \\
\hline no & nucleo-olivary tract. \\
\hline NOT & nucleus of the optic tract \\
\hline ot & optic tract \\
\hline PAG & periaqueductal gray \\
\hline $\mathrm{PC}$ & cerebral peduncle \\
\hline & floccular peduncle \\
\hline PFIdd & dorsal paraflocculus \\
\hline PFIN & ventral parafloceulus \\
\hline $\mathrm{PH}$ & nucleus prepositus hypoglossi \\
\hline PMD & paramedian lobule \\
\hline PO & principal olive \\
\hline $\mathbf{r}$ & AChE-positive cell group $r$ \\
\hline rb & restiform body \\
\hline $\mathrm{rdc}$ & rostral dorsal cap \\
\hline RN & red nucleus \\
\hline sad & dorsal acoustic stria \\
\hline $\mathrm{SC}$ & superior colliculus \\
\hline SI & simple lobule \\
\hline SN & substantia nigra \\
\hline $\mathrm{sV}$ & superior vestibular nucleus \\
\hline $\mathrm{tIV}, \mathrm{t} 4$ & attachment of the roof of the fourth ventricle \\
\hline & ventral lamella of the principal olive \\
\hline vlo & ventrolateral outgrowth \\
\hline VTRL & visual tegmental relay zone \\
\hline $\mathrm{XC}_{1-4}$ & compartments $1-4$ of the nodulus \\
\hline$X Z_{I-Y}$ & zones I-V of the nodulus \\
\hline $\mathrm{y}$ & group y \\
\hline III & oculomotor nucleus \\
\hline V & trigeminal complex \\
\hline VI & abducens nucleus \\
\hline VII & facial nerve \\
\hline VIII & vestibulocochlear nerve \\
\hline
\end{tabular}


their connectivity with different vestibulo-ocular reflexes (Ito et al., 1978, 1982b), and their preferred axes in response to rotating visual patterns (Simpson and Alley, 1974; Simpson et al., 1981; Leonard et al., 1988).

Afferents from the vestibular nuclei do not terminate in the dc or the vlo, but in other subnuclei of the olive including subnucleus $b$ of the caudal MAO, subnucleus $\beta$, and the dmcc (Saint-Cyr and Courville, 1979; Martin et al., 1980; Gerrits et al., 1985; Nelson et al., 1986; Nelson and Mugnaini, 1989; Kaufman et al., 1991). The nucleus prepositus hypoglossi projects both to visual and vestibular subnuclei of the inferior olive (McCrea and Baker, 1985; Gerrits et al., 1985). This projection is partially gammaamino butyric acid (GABA)ergic (De Zeeuw et al., 1993) and partially cholinergic (Barmack et al., 1993). A projection of dorsal group $y$ and the parvicellular subnucleus of the lateral cerebellar nucleus to the vlo and rostral de was described by De Zeeuw et al. (1994). The dc also receives a projection from the pontine paramedian reticular formation (Gerrits and Voogd, 1986).

Azizi and Woodward (1987) included the de and the vlo, together with the subnucleus $\beta$ and subnucleus $c$ in their vertical lamella of the caudal MAO of the rat. The vertical lamella projects to a sagittal zone in the posterior vermis (their zone 4) and the flocculus. They did not recognize the projection in the rat of subnucleus $\mathrm{c}$ to the tecto-olivorecipient zone in the posterior lobe hemisphere (Akaike, $1986 \mathrm{a}, \mathrm{b}, 1987,1992$ ), which is identical to the lateral A zone of Buisseret-Delmas (1988).

A zonal pattern in the olivocerebellar projection to the flocculus, consisting of a central zone from the caudal do and two flanking zones from the rostral dc/vlo, was first demonstrated with autoradiography of axonal transport of tritiated leucine by Groenewegen and Voogd (1977) in the cat. These results were confirmed in a retrograde tracing study in the rabbit (Yamamoto, 1979a) and with anterograde and retrograde axonal transport methods in the cat (Kawamura and Hashikawa, 1979; Sato et al., 1983). The three climbing fiber zones seem to coincide with three Purkinje cell zones that were delineated in the rabbit (Yamamoto and Shimoyama, 1977) and the cat (Sato et al., $1982 \mathrm{a}, \mathrm{b})$ on the basis of their projection to different vestibular and/or cerebellar nuclei. A more complicated pattern with seven climbing fiber zones was described by Gerrits and Voogd (1982) for the olivocerebellar projection to the flocculus of the cat. They identified the caudalmost zone of the flocculus as an extension of the $\mathrm{C}_{2}$ zone, which receives its climbing fibers from the rostral pole of the MAO. Gerrits and Voogd (1982) stressed the extension of some of the floccular zones (including the $\mathrm{C}_{2}$ zone) into the adjoining part of the ventral paraflocculus (the medial extension of the caudal ventral paraflocculus [MEI). The ME differs from the flocculus in receiving an additional projection from the principal olive. In the rabbit a projection of the principal olive to the folium (folium $p$ ) of the ventral paraflocculus that adjoins the flocculus was suggested by Yamamoto (1979a) on the basis of retrograde tracer injections into the ventral paraflocculus including folium $\mathrm{p}$, which may be the equivalent of the ME.

A zonal arrangement in the olivocerebellar projection to the flocculus and the adjacent ventral paraflocculus is also present in the rat (Ruigrok et al., 1992). Five climbing fiber zones were distinguished, including a $\mathrm{C}_{2}$ zone in the caudal flocculus and two pairs of alternating zones, innervated by the caudal de and the rostral dc with the vlo, respectively. All but the most medial zone, which is innervated by the caudal dc, extended into the ventral paraflocculus. Parasagittal zonal patterns in the olivonodular projection were found by several authors; the most detailed pattern was reported by Katayama and Nisimaru (1988) and Balaban and Henry (1988), both in the rabbit.

It is clear that not all studies agree on the location and number of climbing fiber zones in the flocculus. Still, a pattern has emerged of discrete zones (Ito, 1984) or modules (Voogd and Bigaré, 1980) consisting of subsets of Purkinje cells receiving specific climbing fiber input and projecting to distinctive parts of the vestibular and cerebellar nuclei. These modules may constitute the anatomical circuitry used by the vestibulo-ocular (Ito et al., 1977, $1982 \mathrm{~b}$; Sato and Kawasaki, 1990b) and eye movementrelated zones in the flocculus to control eye muscle activity (Dufossé et al., 1977; Balaban, 1984; Sato and Kawasaki, 1984, 1990a, 1991; Nagao et al., 1985). An intrinsic reference revealing these zones, such as the one provided by the acetylcholinesterase ( $\mathrm{AChE}$ )-positive raphes that subdivide the white matter of the cerebellum (Hess and Voogd, 1986; Voogd et al., 1987), has never been used to correlate anatomical and physiological data. Such a reference became available with our recent use of $\mathrm{AChE}$ histochemistry to subdivide the white matter of the rabbit flocculus into five compartments (Tan et al., 1995). The present study addresses the following questions: do the afferent climbing fibers from different parts of the dc/vlo complex travel in specific white matter compartments in the flocculus and the nodulus; and what is the relationship between the white matter compartments and the climbing fiber termination zones?

\section{MATERIALS AND METHODS}

Twenty pigmented, Dutch belted rabbits were anesthetized by an initial intramuscular injection of a mixture of ketamine $(32 \mathrm{mg} / \mathrm{kg})$, acepromazine $(0.32 \mathrm{mg} / \mathrm{kg})$, and xylazine $(5 \mathrm{mg} / \mathrm{kg})$. Supplements were given every $30-45$ minutes $(9 \mathrm{mg} / \mathrm{kg}$ ketamine, $0.09 \mathrm{mg} / \mathrm{kg}$ acepromazine, 2 $\mathrm{mg} / \mathrm{kg}$ xylazine $)$. Small quantities $(5-15 \mathrm{nl})$ of a $7 \%$ solution in physiological saline of horseradish peroxidase coupled to wheat germ agglutinin (WGA-HRP, Sigma type VI) were injected with air pressure applied to a micropipette placed in the inferior olive. Nine of these cases were selected for detailed study. The sites for injection were located by electrophysiological recording, using the method and criteria of Leonard et al. (1988) for assessing the responses of $\mathrm{dc} /$ vlo neurons to rotatory visual stimuli. A dorsal approach through the fourth ventricle avoided the cerebellum. Recordings and injections were made through the same glass micropipette filled with the WGA-HRP solution. Micropipettes were fitted with a tungsten wire, etched to a thin point. Tip diameter of the micropipettes was $20 \mu \mathrm{m}$.

Neurons in the caudal dc can be recognized by their preference for rotation of the visual surround about the vertical axis; rostral dc/vlo neurons prefer rotations about specific horizonal axes. Eye dominance can also be used to distinguish caudal dc and rostral dc/vlo responses during visual stimulation. Flash stimuli to the ipsilateral and contralateral eye were used to determine the relative rostrocaudal position of the tip of the injection pipette. Flash potentials from caudal dc neurons were optimal when the stimulus was delivered to the contralateral eye. Rostral dc neurons responded optimally with flash stimulus to the 
ipsilateral eye, while vlo neurons and the most rostral neurons in the rostral dc showed hardly any response to the flash stimulation.

After a survival time of $36-48$ hours the rabbits were deeply anesthetized and perfused transcardially with 1 liter of saline at room temperature, followed by 2 liters of a solution containing 1-3\% paraformaldehyde and $0.5-1.25 \%$

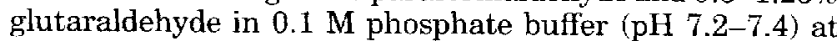
room temperature, and finally with 1 liter of $10 \%$ sucrose in $0.1 \mathrm{M}$ phosphate buffer ( $\mathrm{pH} 7.2-7.4$ ) at $4^{\circ} \mathrm{C}$. The brainstem and cerebellum were removed, rinsed in $10 \%$ sucrose phosphate buffer, and embedded in a solution of $10 \%$ gelatin and $10 \%$ sucrose. The embedded tissue was stored overnight in $30 \%$ sucrose in $0.1 \mathrm{M}$ phosphate buffer ( $\mathrm{pH}$ $7.2-7.4)$ and then cut into $40-\mu \mathrm{m}$ transverse sections. Adjacent serial sections were processed to obtain four 1-in-4 series as follows: with diaminobenzidine (DAB; Graham and Karnovsky, 1966) as a chromogen in the histochemical reaction for horseradish peroxidase; with tetramethylbenzidine (TMB; Gibson et al., 1984) as a chromogen; reacted for AChE; and Nissl stained. A standard thiocholine method (Geneser-Jensen and Blackstad, 1971) was used with acetylthiocholine iodide as a substrate and ethopropazine as a nonspecific AChE blocker (Hess and Voogd, 1986). The extent of the injection site was assessed in DAB-reacted sections with light microscopy. The climbing fibers in the white matter and molecular layer were plotted from the TMB-reacted sections and their position was compared with the AChE-stained raphes in adjacent sections. The serial DAB- and TMB-processed sections of one case with an injection of WGA-HRP in the rostral MAO (B 2202) were put at our disposal by Drs. C. Yeo and J.M. van Ham, from the neurobiology group of the Department of Anatomy of University College (London, U.K.).

In three rabbits (C 914, C 1134, and C 1135) tritiated leucine was injected in the caudal inferior olive. A stock solution of $\mathrm{L}\left[4,5^{3} \mathrm{H}\right]$ leucine (specific activity about 140 $\mathrm{Ci} / \mathrm{mM}$, concentration $1 \mu \mathrm{Ci} / \mu \mathrm{l}$; Radiochemical Centre, Amersham, UK) was evaporated to dryness under a gentle flow of nitrogen at $40^{\circ} \mathrm{C}$ and redissolved in saline to a final concentration of $75 \mu \mathrm{Ci} / \mu \mathrm{l}$. Injections were performed with a 1 - $\mu$ l Hamilton syringe using a 25 -gauge needle. To reduce unwanted spread of $\left[{ }^{3} \mathrm{H}\right]$ leucine, delivery started 5 minutes after placing the needle in position. The injection was made at a rate of $0.1 \mu \mathrm{l} / 10 \mathrm{~min}$. After the injection the needle was left in place for an additional 15 minutes. Following a survival time of 4-7 days the animal was transcardially perfused under deep anesthesia with 0.5 liter of saline and 2 liters of $4 \%$ formaldehyde. The brainstem and cerebellum were removed and embedded in $10 \%$ gelatin and transversely sectioned at $30 \mu \mathrm{m}$ on a freezing microtome. Sections were mounted on slides prepared with a chromealum gelatin solution, defatted in xylene, dipped in Ilford $\mathrm{G} 5$ emulsion, and exposed for 6 weeks at $4^{\circ} \mathrm{C}$. The sections then were developed in Kodak D19 at $16-18^{\circ} \mathrm{C}$ for 4 minutes, rinsed in distilled water, fixed in $24 \%$ sodium thiosulphate for 8 minutes, and counterstained with cresyl violet. Criteria previously described by Groenewegen and Voogd (1977) were used to delineate the effective injection site.

All injection sites were plotted in serial transverse sections of the inferior olive. Injection sites in all experiments are illustrated in selected sections and in graphical reconstructions of the inferior olive, prepared according to Brodal (1940) and Gerrits and Voogd (1982). The caudal dc and the rostral dc are separated by a narrow neck (Leonard et al., 1988). 'The border between the rostral dc and the vlo is arbitrary. The vlo is continuous with the dorsal leaf of the principal olive.

The resulting labeling of the climbing fibers was represented in diagrams of the unfolded Purkinje cell layer of the flocculus and the adjacent ventral paraflocculus. The Purkinje cell layer in each transverse section was unfolded as a series of straight line segments approximating the contour of the folia, and the portions of the molecular layer containing labeled climbing fibers were demarcated (Fig. 1A,B). The sections were aligned using the distance between the midline and the most ventromedial point of the Purkinje cell layer of the flocculus (point B in Fig. 1). The distance between the sections was multiplied by the magnification factor, and the points on the lines representing borders between folia and between climbing fiber zones were interconnected (Fig. 1C).

The folial rosette of the flocculus usually consists of four or five folia that fuse caudally. They are indicated from ventral to dorsal as folium $m$ and $f 1-4$. The cortices of the flocculus and the adjoining folium of the ventral paraflocculus (folium $p$ of Yamamoto and Shimoyama, 1977) are continuous in the bottom of the posterolateral fissure. The compartments of the white matter of the flocculus are called (from lateral to medial) $\mathrm{C}_{2}$ and $\mathrm{FC}_{1-4}$. The $\mathrm{AChE}$ positive borders of the compartments (the raphes) are indicated by the numbers of the compartments they separate (e.g., 1/2). Climbing fiber zones are indicated with the prefix FZ (floccular zone) and the Roman numeral of the corresponding compartment. Compartments in AChEstained sections containing the nodulus (lobule $X$ ), and the corresponding climbing fiber zones, are indicated as $\mathrm{XC}_{1-4}$ and $X Z_{I-V}$, respectively. The distribution of retrograde labeling in the AOS nuclei and VTRZ in experiments with injections of WGA-HRP into the inferior olive was determined and compared with the rostrocaudal extent of the injection site in the dc and the vlo (Fig. 10).

\section{RESULTS \\ Projections of the caudal dorsal cap (caudal de)}

In six rabbits the targeted injection site was the caudal half of the dorsal cap (caudal dc). In four cases ( $\mathrm{K} 324, \mathrm{~K}$ $369, \mathrm{~K} 426$, and $\mathrm{C} 914$ ) the injection site did not extend beyond the narrow neck separating the caudal dc from the rostral dorsal cap (rostral de). In two other cases ( $\mathrm{K} 314$ and $K$ 325) the injection site included the caudal part of the rostral dc. One experiment out of each of these two groups will be described in some detail.

The injection site in case K 369 (Fig. 2A) includes, in addition to the caudal dc, the medial part of the caudal MAO and subnucleus $\beta$. Retrogradely labeled neurons in the AOS are limited to the NOT, the DTN, and ITN (Fig. 10A). Plots from serial sections showing the course and termination of the labeled olivocerebellar fibers and diagrams of the borders of the compartments of the flocculus and the nodulus from adjacent AChE-stained sections are illustrated in Figure 3. The labeled climbing fiber zones in this experiment are reconstructed for the flocculus in Figure 2B and for the nodulus in Figure 7a. Fibers from the caudal dc cross the midline, pass lateral to the spinal tract of the trigeminal nerve, and accumulate along the medial and dorsal border of the restiform body (Fig. 3e). Many 

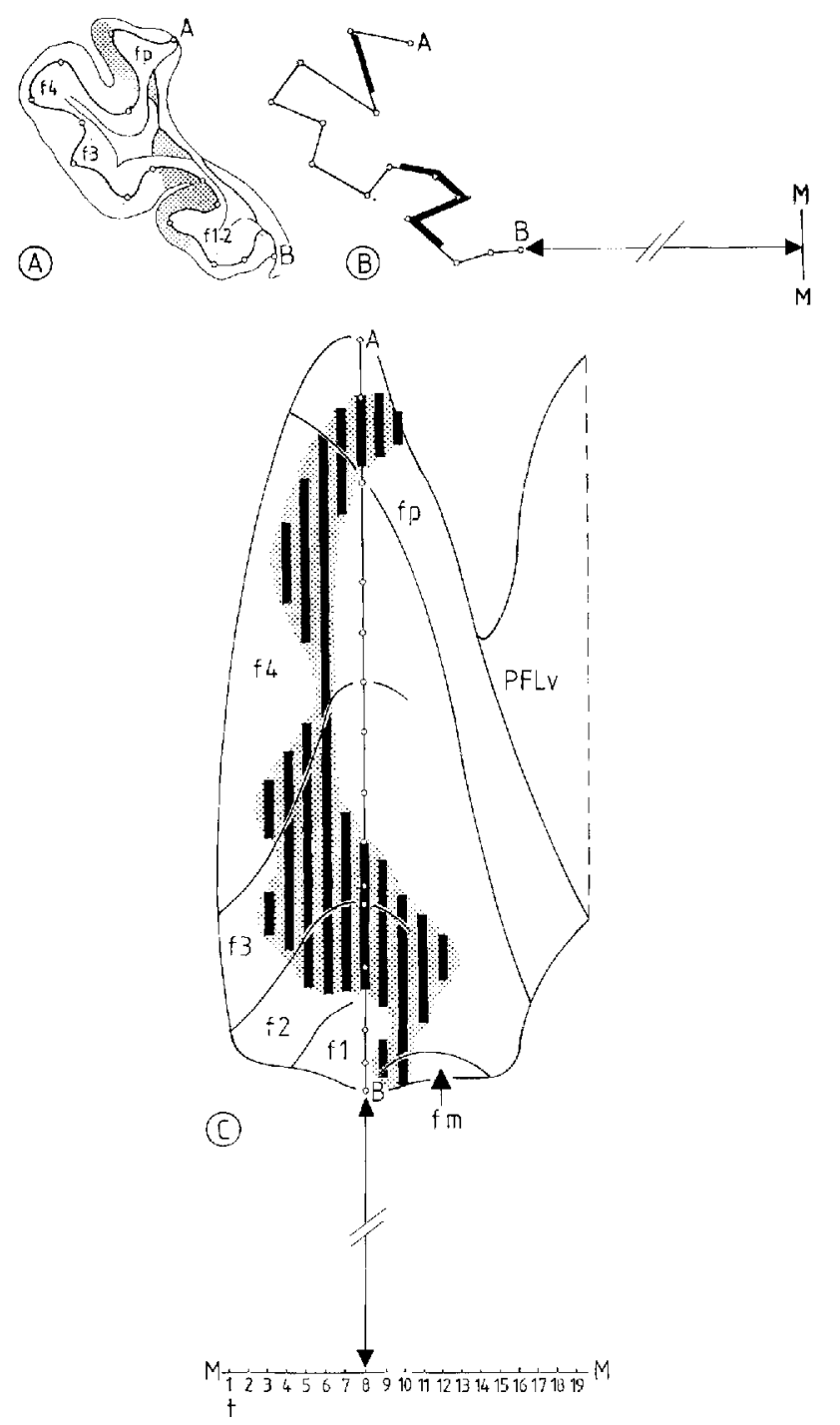

Fig. 1. Diagram illustrating the unfolding of the Purkinje cell layer used to prepare maps of the unfolded flocculus. The Purkinje cell layer A-B was straightened, and the position of labeled climbing fibers (in this case in $\left.\mathbf{F Z}_{\mathrm{II}}\right)$ was indicated (A,B). Sections were aligned using the distance between the midline (M) and the most ventromedial point of the Purkinje cell layer (B). The position of the section illustrated in A and $B$, and also the $\mathrm{FZ}_{\mathrm{II}}$ zone, is indicated on the reconstructed map of the flocculus (C). The map is positioned as if viewed from the lateral side. The distance between the reconstructed sections $t$ was obtained by multiplying the true distance between the sections by the magnification factor and, therefore, the rostrocaudal scale is the same as the dorsoventral scale.

labeled fibers enter the cerebellum along the superior cerebellar peduncle. Within the cerebellum they pass rostral and dorsal to and through the anterior interposed nucleus to be distributed to the white matter of the vermis and the medial part of the hemisphere (Fig. $3 \mathrm{~b}-\mathrm{g}$ ). Labeled fibers from the caudal dc enter the floccular white matter medially. Some of these fibers arch through or around the parvicellular lateral cerebellar nucleus (Lpc). Within the flocculus they separate into two streams. One stream passes through the medial white matter of the flocculus (Fig. 3d) to accumulate in a bundle that occupies an area corresponding to the $\mathrm{FC}_{2}$ compartment as delineated in adjacent AChE-stained sections (Figs. 3a-e, 4, 5, 6). The fibers of the second stream accumulate in compartment $\mathrm{FC}_{4}$ (Figs. 3a,b, 4, 5). Within $\mathrm{FC}_{2}$ the fibers are densely packed, especially medially. Their distribution in $\mathrm{FC}_{2}$ is complementary to the $\mathrm{AChE}$ staining, which is more concentrated in the lateral part of this compartment (Fig. 6D). From this compartment labeled fibers enter the granular layer to terminate as climbing fibers in a continuous strip in the molecular layer of folia $\mathrm{f}^{1-4}$, including the rostral pole of folium $p$ (Figs. 2B, 3a-d, 5, 6). The border between labeled and unlabeled parts of the cortical layers is sharp. Climbing fiber zone $\mathrm{FZ}_{\mathrm{II}}$ gradually shifts laterally in more rostral sections.

Labeled fibers of the second fiber stream accumulate at the border of the white matter of the flocculus within the restiform body and the brachium pontis along the AChEpositive cell strands in this region (Fig. 3c,d). Where the raphe between $\mathrm{FC}_{3}$ and $\mathrm{FC}_{4}$ can be clearly distinguished in the rostral flocculus they become located in $\mathrm{FC}_{4}$, where they travel in a rostrodorsal direction (Fig. $3 \mathrm{a}-\mathrm{c}, 5 \mathrm{~A}, \mathrm{C}$ ). They terminate as the climbing fiber zone $\mathrm{FZ}_{\mathrm{IV}}$ in the medial parts of folia 3 and 4 (Fig. $2 B$ ). $\mathrm{FZ}_{\mathrm{IV}}$ does not extend into folium $\mathrm{p}$. $\mathrm{FZ}_{\mathrm{II}}$ and $\mathrm{FZ}_{\mathrm{IV}}$ are separated from each other by an empty strip of molecular layer corresponding to $\mathrm{FZ}_{\mathrm{III}}$, which is innervated by the rostral dc and the vlo through $\mathrm{FC}_{3}$ (see below). Labeled climbing fibers from this injection site also terminate in isolated patches in caudal folium $\mathrm{p}$ without a clear zonal pattern. This labeling cannot be associated with a specific compartment or climbing fiber zone (Fig. 2B).

Labeled olivocerebellar fibers in $\mathrm{FC}_{2}$ can be traced caudally in the roof of the lateral recess, ventral to the lateral cerebellar nucleus. They continue their course in the roof of the fourth ventricle, near the attachment of the posterior medullary velum, to enter the nodulus at its lateral side (Fig. 3e,f). Here they terminate in two climbing fiber strips, one in the medial and one in the lateral part of this lobule.

Four compartments can be distinguished in the white matter of the nodulus in adjacent $\mathrm{AChE}$-stained sections (Figs. 3f,g, 9F). Rather wide compartments $\mathrm{XC}_{1}$ and $\mathrm{XC}_{2}$ are present in the medial two-thirds of the lobule. A distinct $\mathrm{AChE}$-positive raphe delineates $\mathrm{XC}_{3}$, which is only present in the dorsal white matter of the nodulus (Fig. 9A,E,F). It corresponds to a similar, but wider compartment in the lateral white matter of the uvula. Compartment $\mathrm{XC}_{4}$ is narrow and occupies the lateral white matter of the nodulus. Labeled climbing fibers are located in $\mathrm{XC}_{1}$ and $\mathrm{X}_{4}$ (Fig. $9 F, G)$. Those contained in $\mathrm{XC}_{4}$ terminate in two concentrations of climbing fibers on the ventrolateral aspect of lobule $\mathrm{X}$ and in a narrow single strip on the dorsal aspect of the lobule $\left(\mathrm{XZ}_{\mathrm{TV}}\right.$, Fig. 7a). Labeled fibers in $\mathrm{XC}_{1}$ innervate the $\mathrm{XZ}_{\uparrow}$ zone, flanking the midline. This zone continues from the ventral side of the lobule onto its dorsal side and farther into lobule IX. A rostrolateral triangular area, indicated with a question mark in Figure $7 \mathrm{a}$, is clearly separated from the rest of $X Z_{I}$. In all of our experiments the injection site included both the caudal dc and the subnucleus $\beta$. These experiments, therefore, do not distinguish between medial and lateral subzones of zone $\mathrm{XZ}_{\mathrm{I}}$ innervated by subnucleus $\beta$ and caudal dc, respectively (Katayama and Nisimaru, 1988).

Other fibers head for the midline dorsal to the cerebellar nuclei and constitute a parasagittal band of labeled fibers in the molecular layer of the vermis close to the midline. They terminate in the medial A zone of Groenewegen and Voogd 


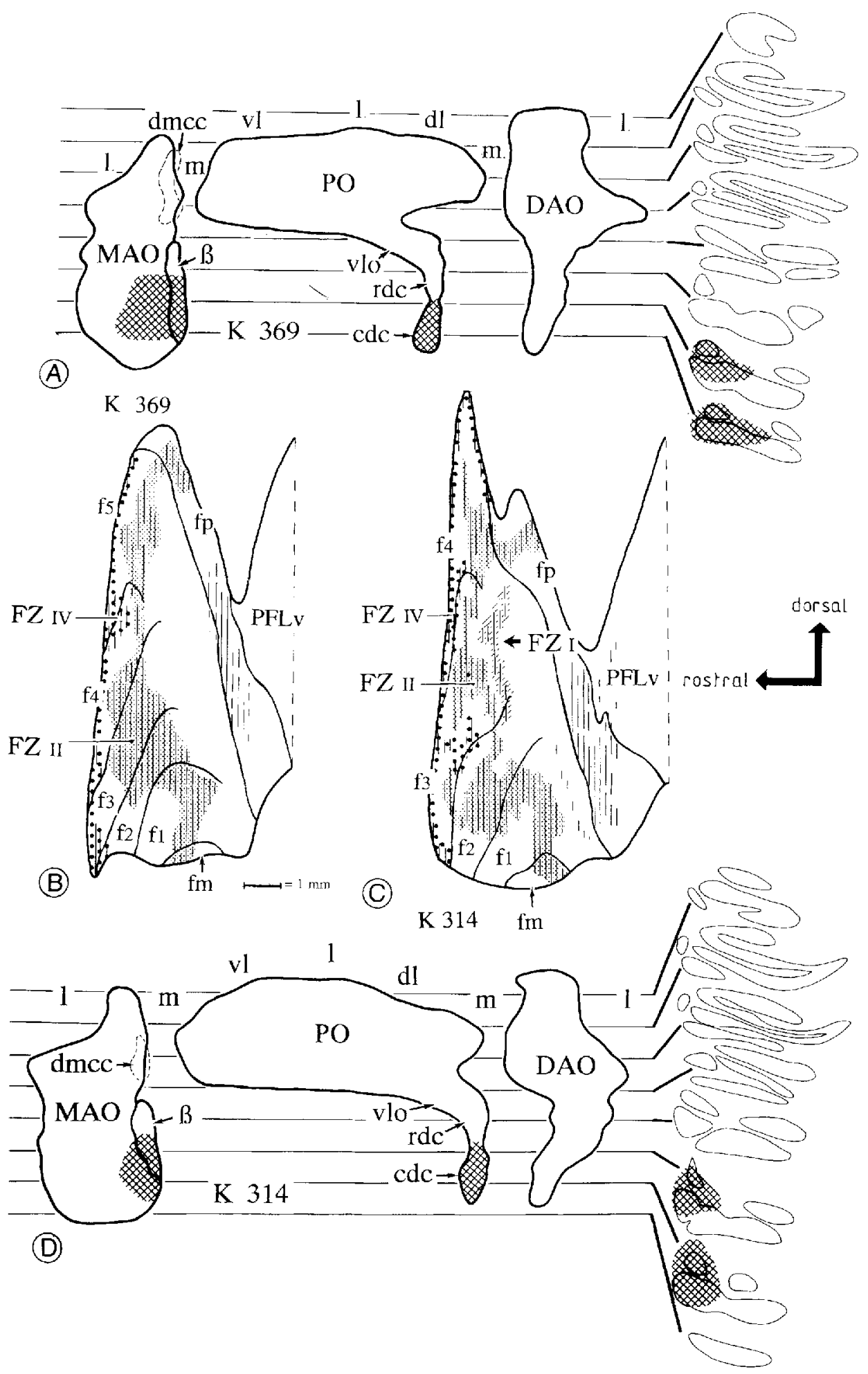

Fig. 2. A-D: Drawings of the distribution of labeled climbing fibers in the unfolded flocculus and ventral paraflocculus and the injection site in the caudal dorsal cap. The presence of labeled climbing fibers in the sections used to prepare the unfolded flocculus is indicated by vertical lines in $B$ and $C$. The estimated extent of the $\mathrm{FZ}_{\mathrm{TV}}$ zone is indicated by large dots while that of the $\mathrm{FZ}_{\mathrm{II}}$ zone is indicated by small

dots. Climbing fibers in caudal folium $p$ cannot be associated with these zones. In case $\mathrm{K} 314$ some climbing fibers also terminated in $\mathrm{FZ}_{\mathrm{I}}$ (oblique hatching: arrow). Injection sites are indicated in a diagram of the unfolded inferior olive and in assorted transverse sections. A and $B$ : case $\mathrm{K} 369$; $\mathrm{C}$ and D: case $\mathrm{K}$ 314. Scale in B also applies to C, and the rostrocaudal scale is the same as the dorsoventral scale. 

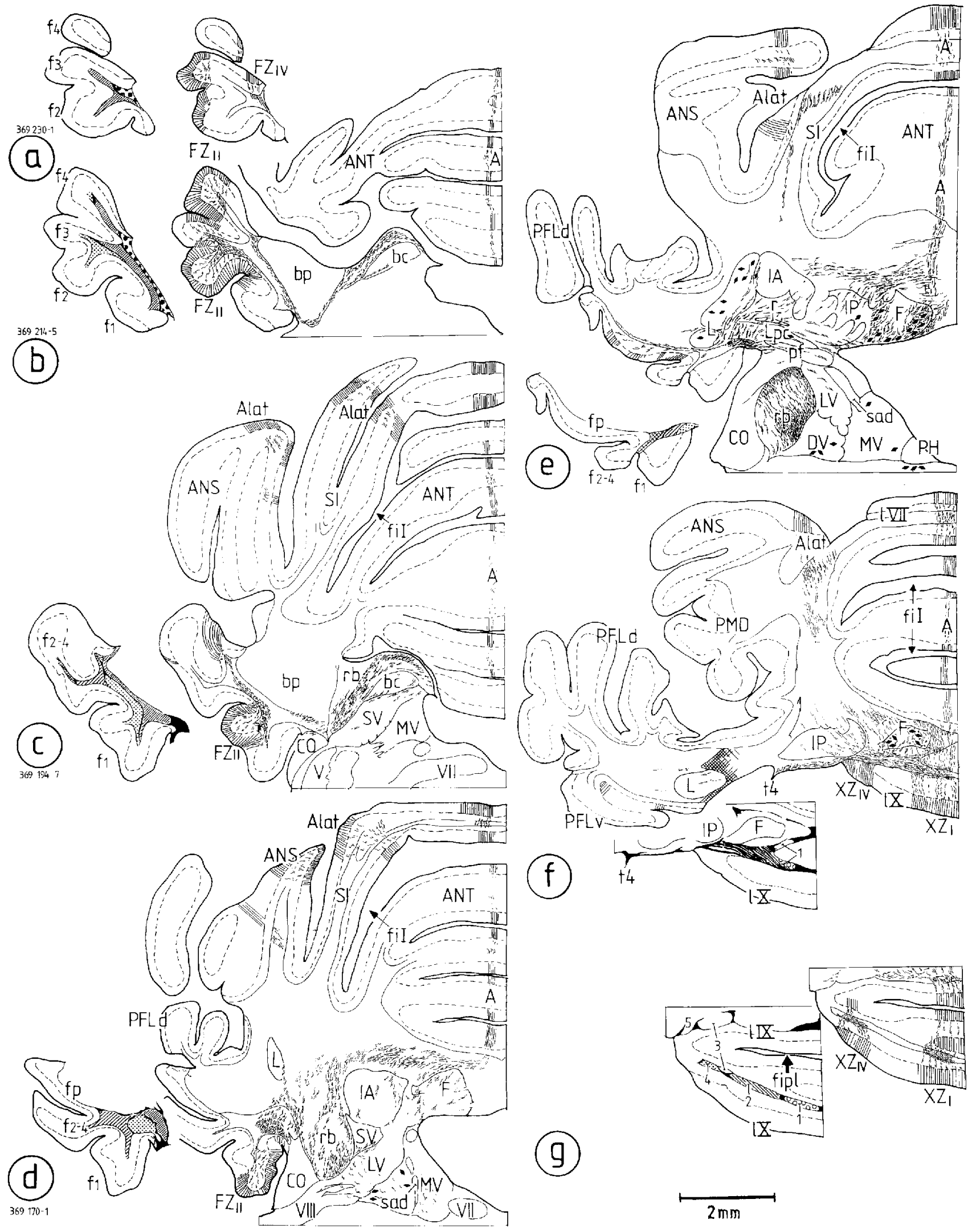

Fig. 3. a-g: Drawings of acetylcholinesterase (AChE)-stained sections through the flocculus and the caudal vermis (left columns) and of adjacent sections incubated for horseradish peroxidase (HRP) through the flocculus and the cerebellum (right columns) in case K 369 . Note the presence of labeled olivocerebellar fibers in the $\mathrm{FC}_{2}$ and $\mathrm{FC}_{4}$ compartments of the flocculus and the $\mathrm{XC}_{1}$ and $\mathrm{XC}_{4}$ compartments of the

nodulus and the termination of these fibers in the corresponding zones. White matter compartments $\mathrm{XC}_{1-4}$ of the nodulus are indicated with arabic numerals $1-4$. In addition, olivocerebellar fibers terminate in the A zone of the anterior vermis (A) and in the lateral A zone (Alat) of Buisseret-Delmas (1988). (Compartments and zones designated as in Fig. 4.) 


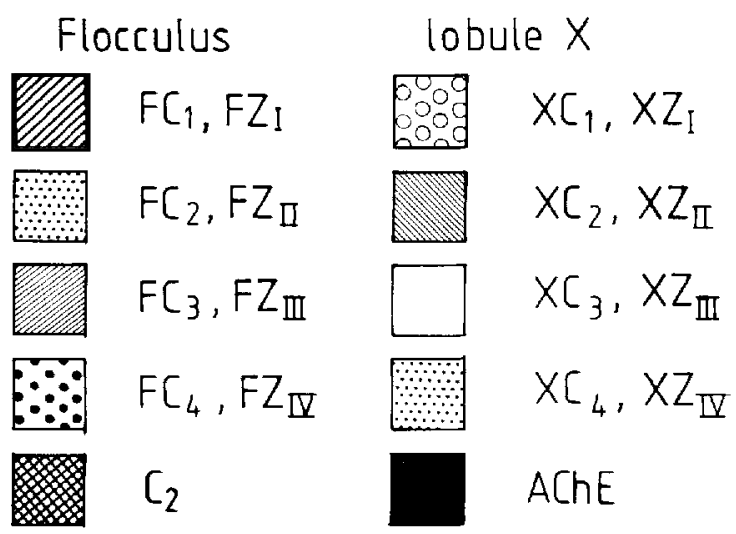

Fig. 4. Explanation of the symbols used to indicate compartments and zones in the floceulus and the nodulus in Figures 2, 3, 11, 13, 18, and 20 .

(1977). Other bundles of labeled olivocerebellar fibers enter the white matter of the paramedian, ansiform, and simple lobules from the region of the dorsolateral protuberance of the fastigial nucleus. They terminate as a continuous climbing fiber zone in the medial part of the molecular layer of these lobules (Alat in Fig. 3b-f) that corresponds to the lateral A zone of Buisseret-Delmas (1988) in the rat. Labeled climbing fibers are present in both the medial and the lateral A zone of the simple lobule (Fig. 3b-f). 'The two climbing fiber zones are discontinuous and remain separated by an empty area of molecular layer, which contains climbing fibers of the $B$ zone in other experiments. The medial A zone and the lateral A zone are innervated from the subnuclei $b$ and $c$ of the caudal MAO, respectively, and they are included in the injection site in this case.

Retrogradely labeled cells are present mainly in the medial part of the fastigial nucleus. A few labeled cells are located in the lateral cerebellar nucleus. Retrogradely labeled cells in the vestibular complex are found in the caudal descending vestibular nucleus (DV) bilaterally and in the ventral part of the ipsilateral magnocellular medial vestibu lar nucleus (MVmc). Very few labeled neurons are present in the superior vestibular nucleus (SV), and none are located in group $y$ or in the parvicellular extension of the lateral cerebellar nucleus.

Similar observations were made on the distribution of $\left[{ }^{3} \mathrm{H}\right]$ leucine-labeled climbing fibers in the nodulus and the flocculus in case $\mathrm{C} 914$, with an injection of the caudal dc, which may have included part of subnucleus $\beta$ (Fig. 15A).

The injection site in case $\mathrm{K} 314$ includes the caudal MAO, caudal DAO, subnucleus $\beta$, caudal dc, and in addition a bit of the caudal part of the rostral de (Fig. 2D). The distribution of retrogradely labeled neurons in the AOS nuclei is similar to cases $\mathrm{K} 369$ and $\mathrm{K} 426$, but in addition a small number of weakly labeled neurons was observed in the VTRZ. Labeled fibers are located in compartments $\mathrm{FC}_{2}$ and $\mathrm{FC}_{4}$ and terminate in the corresponding climbing fiber zones $\mathbf{F Z}_{\mathrm{II}}$ and $\mathrm{FZ}_{\mathrm{IV}}$ (Fig. 12A,B). In this case a third fiber stream is present. It separates from the second fiber stream in the caudal flocculus and travels in $\mathrm{FC}_{1}$ in a lateral direction toward folium 2 and 3 to terminate in the most lateral parts of the molecular layer of these folia. In the reconstruction of Figure $2 \mathrm{C}$ it is indicated as $\mathrm{FZ}_{\mathrm{I}}$. The distribution of labeling in the nodulus is similar to the previous cases (Fig. 7b). Climbing fiber labeling was present in the medial A and the $\mathrm{B}$ zone of the anterior vermis and in the lateral A zone of Buisseret-Delmas (1988) in the hemisphere of the posterior lobe. Labeled climbing fibers of the $\mathrm{B}$ zone clearly separate labeling in the medial and lateral A zones in the simple lobule.

\section{Projection of the rostral dorsal cap and ventrolateral outgrowth (rostral dc/vlo)}

In five rabbits ( $\mathrm{K} 327, \mathrm{~K} 328, \mathrm{~K} 333, \mathrm{~K} 397$, and $\mathrm{C} 1135$ ) the injection site involved the rostral dc/vlo. Three of these cases (K 328, K 397, and C 1135) will be presented. Labeling in the flocculus was found in zones and compartments that interleaved with those found with injections of the caudal dc. Labeling in the nodulus occupied an intermediate position.

The injection site in case $\mathrm{K} 328$ is limited to the rostral de and vlo (Fig. 11A). Retrogradely labeled neurons in the AOS nuclei are only present in the VTRZ (Fig. 10B). Plots of the labeled fibers and the compartments in the flocculus and the nodulus are illustrated in Figure 13. The zonal distribution of climbing fibers in the flocculus and the nodulus is shown in Figures $7 \mathrm{~d}$ and 11B. Labeled olivocerebellar fibers, located along the dorsolateral border of the restiform body, enter the white matter of the flocculus. Some labeled fibers ramify among the cells of group y (Fig. 13d). Labeled fibers occupy compartment $\mathrm{FC}_{3}$ and, arching over $\mathrm{FC}_{2}$, also distribute to compartment $\mathrm{FC}_{1}$. They can be traced through $\mathrm{FC}_{1}$ and $\mathrm{FC}_{3}$ to terminate as climbing fibers in the $\mathrm{FZ}_{\mathrm{I}}$ and $\mathrm{FZ}_{\text {III }}$ zones (Figs. 11B, 13a-d). FZ I occupies a lateral position, but remains separated from the lateral margin of the flocculus by an unlabeled stretch of molecular layer, corresponding to the $\mathrm{C}_{2}$ zone (see below), which broadens more caudally. The $\mathrm{C}_{2}$ compartment of the flocculus does not contain any labeled fibers (Fig. $13 \mathrm{c}, \mathrm{d}$ ). $\mathrm{FZ}_{\mathrm{II}}$ is separated from $\mathrm{FZ}_{\mathrm{T}}$ by an empty strip, corresponding to $\mathrm{FZ}_{\mathrm{II}}$ (Fig. 13a-c). $\mathrm{FZ}_{\mathrm{T}}$ and $\mathrm{FZ}_{\mathrm{TII}}$ extend into folium $\mathrm{p}$, where they appear to merge. Patchy climbing fiber labeling that cannot be associated with either zone is present in the molecular layer of the central part of folium $\mathrm{p}$ (Figs. 11B, 13c,d).

Labeling in compartment $\mathrm{FC}_{3}$ can be traced caudally, ventral to the lateral cerebellar nucleus and medially along the attachment of the roof of the fourth ventricle (Fig. 13e). These fibers subsequently enter the nodulus. Ilere they occupy compartments $\mathrm{XC}_{2}$ and $\mathrm{XC}_{4}$, which can be delineated in adjacent AChE-stained sections (Figs. 9C,D, 13f). Fibers from $\mathrm{XC}_{2}$ terminate as climbing fibers in the $\mathrm{XZ}_{\mathrm{II}}$ zone (Fig. 7d), which is continuous in the bottom of the posterolateral fissure with a similar zone in lobule IX. Labeled fibers in compartment $\mathrm{FC}_{4}$ terminate in a narrow lateral strip on the ventral and dorsal aspect of this lobule, indicated as $\mathrm{XZ}_{\mathrm{IV}^{*}}$ in Figure $7 \mathrm{~d}$. The medial compartment $\mathrm{XC}_{1}$ and the medial cortex do not contain labeled fibers.

A heavy concentration of retrogradely labeled cells is present in the Lpc (Fig. 13d), and a few labeled cells are found in the fastigial nucleus. Neurons of dorsal group $y$, lying within the floccular peduncle or of ventral group $y$, capping the restiform body are not labeled. ${ }^{1}$ Labeled fibers pass medially from the $L p c$ in the rostral wall of the lateral

Identical retrograde labeling after injections of WGA-HRP in the contralateral vlo and rostral de was reported by De Zeeuw et al (1994). They concluded that not only the Lpc, but also the dorsal part of dorsal group $y$ contained retrogradely labeled small to medium-sized neurons. However, they ineluded the ventral part of the Lpc, which contains some large oval neurons that project to the contralateral oculomotor nucleus (Highstein and Reisine, 1979), in dorsal group y. 

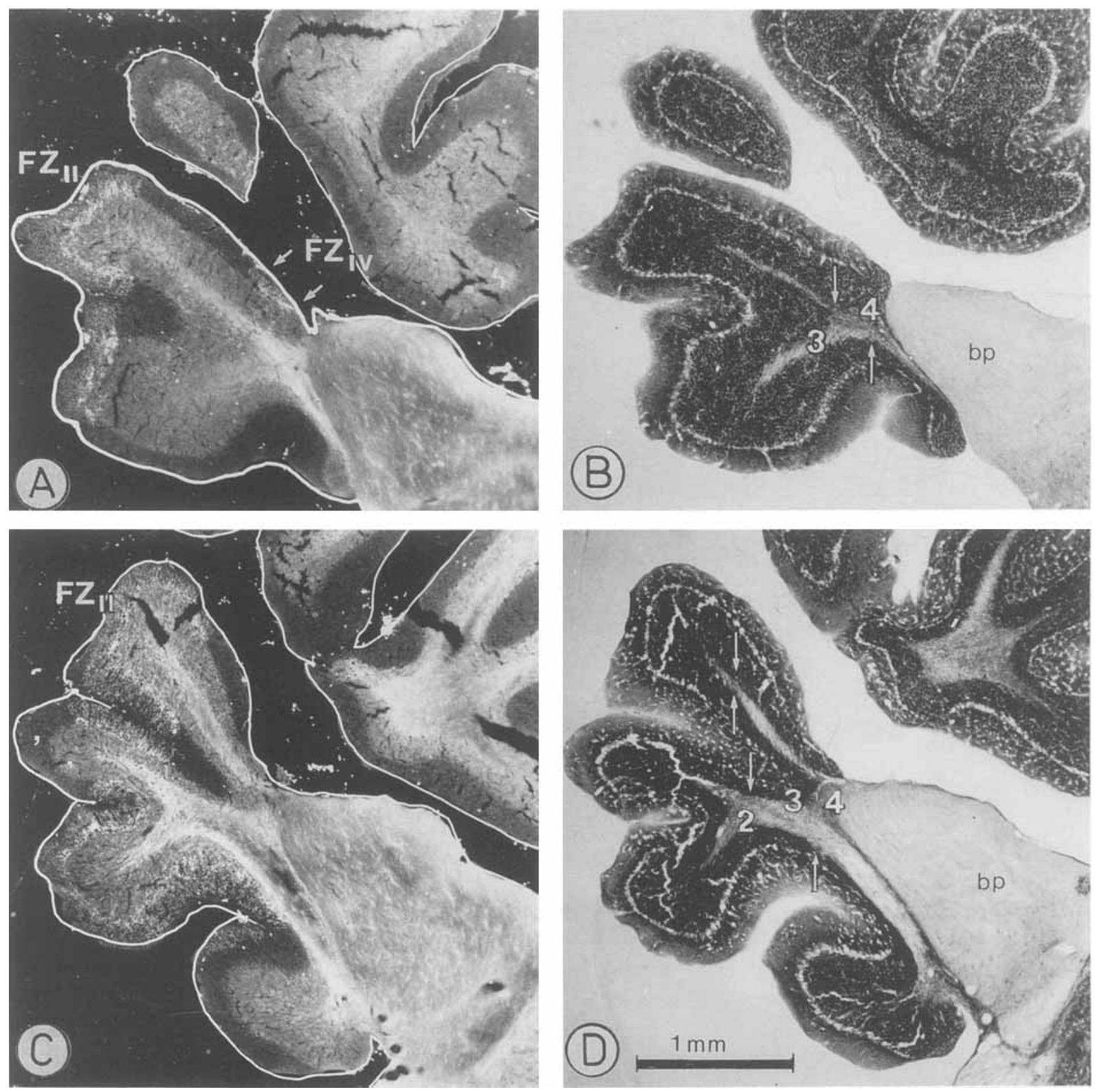

Fig. 5. Darkfield photographs of two HRP-incubated sections through the flocculus in case K 369 (A, rostral; $\mathbf{C}$, caudal) and brightfield photomicrographs of adjacent $\mathrm{AChE}$-stained sections $(\mathbf{B}, \mathbf{D})$. Small white arrows indicate $\mathrm{AChE}$-positive raphes. Compartments $\mathrm{FC}_{1-4}$ are indicated by arabic numerals.

recess and the lateral angle of the fourth ventricle (no, Fig. $13 \mathrm{~d})$. They ascend in a bundle located ventral and ventrolateral to the superior cerebellar peduncle (Fig. $13 a-c)$, which enters the decussation of the brachium conjunctivum. Their localization corresponds to the nucleo-olivary fibers (Legendre and Courville, 1987), which were retrogradely labeled in this experiment.

The injection site in K 397 is larger than that in K 328 and in addition to the rostral dc/vlo, it also involves the dmcc and rostromedial parts of the DAO and MAO, except for their rostralmost poles (Fig. 11D). Retrogradely labeled cells are present in the VTRZ, but they were absent from the NOT, DTN, and ITN. Hardly any labeled cells were found in the MTN and lateral terminal nucleus (LTN). The distribution of labeled olivocerebellar fibers in the flocculus is similar to the previous case (Fig. 11C). The nodulus contains labeled fibers in compartment $\mathrm{XC}_{3}$, terminating in the $\mathrm{XZ}_{\mathrm{III}}$ on the dorsal surface of the lobule, in addition to the labeling of $\mathrm{XZ}_{\mathrm{II}}$ (Figs. 7e, 9B), which was also present in K 328 (Figs. 7d, 9D). The climbing fibers terminating in $\mathrm{XZ}_{\mathrm{III}}$ probably originate from the dmcc, which was included in the injection site.

Extensive retrograde labeling is present among neurons of the cerebellar nuclei, including the Lpc (Fig. 14D). The neurons of dorsal group $y$ (located between the fibers of the floccular peduncle) and of ventral group y (located ventral to the peduncle and dorsal to the restiform body) are not labeled. The nucleo-olivary fiber bundle situated in the lateral recess of 

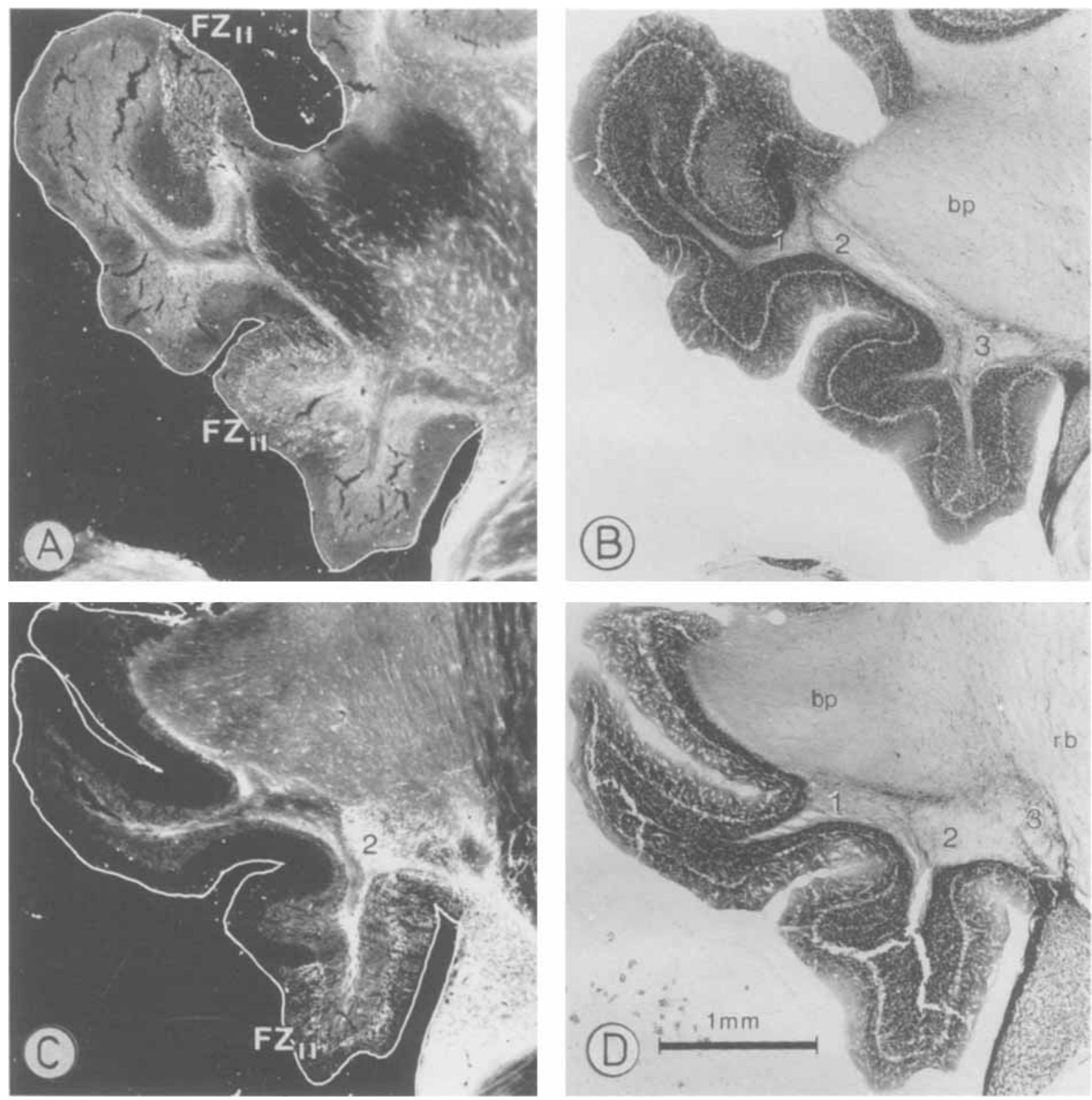

Fig. 6. Darkfield photographs of two HRP-incubated sections (A, C) and adjacent AChE-stained (B, D) sections from case K 369. A, B: Rostral level. C, D: Caudal level.

the fourth ventricle and ventral to the superior cerebellar peduncle is heavily labeled (no, Fig. 14B,D).

In one case (C 1135, Fig. 15B) an injection of tritiated leucine was made in the vlo, but it also extended widely into other parts of the inferior olive, including the rostral pole of the MAO, which is thought to contain neurons projecting to the $\mathrm{C}_{2}$ zone, and the dmcc. In the rostral dc/vlo the injection site was situated somewhat more rostrally than in the previous two cases, $\mathrm{K} 328$ and $\mathrm{K} 397$. The distribution of fibers in the floccular cortex is similar except for the presence of labeled climbing fibers in the cortex lateral and caudal to $\mathrm{FZ}_{\mathrm{I}}$, corresponding to the $\mathrm{C}_{2}$ zone, which was unlabeled in $\mathrm{K} 328$ and $\mathrm{K} 397$. In $\mathrm{C} 1135$ labeling in the $\mathrm{C}_{2}$ zone continues into the medial part of the ventral paraflocculus and the dorsomedial and lateral part of the dorsal paraflocculus. Some labeled fibers are present in the fastigial and posterior interposed nucleus. Most are located in and dorsal to the anterior interposed and the medial part of the lateral cerebellar nucleus.

The white matter of the nodulus in $\mathrm{C} 1135$ contains bundles of labeled fibers in an intermediate and a lateral position. They terminate as climbing fibers in a central zone corresponding in position to $\mathrm{XZ}_{\mathrm{II}}$ of the previous cases. In addition, an extremely lateral zone of molecular layer surrounding the lateral pole of the lobule (indicated as $\mathrm{XZ}_{\mathrm{V}}$ in Fig. 16A) and a strip on the dorsal surface of the lobule $\left(\mathrm{XZ}_{\mathrm{III}}\right)$ contain labeled climbing fibers. These fibers presumably take their origin from the rostral MAO and the dmcc. Regions of the molecular layer corresponding to $\mathrm{XZ}_{\mathrm{I}}$ and $\mathrm{XZ}_{\mathrm{IV}}$ remain virtually empty (Figs. $7 \mathrm{f}, 16 \mathrm{~A}$ ). 

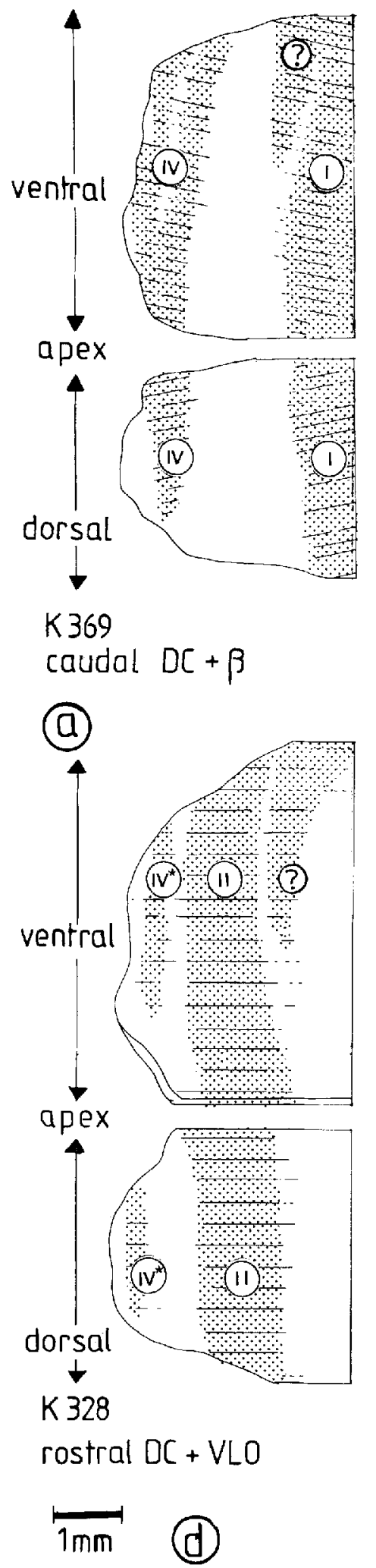

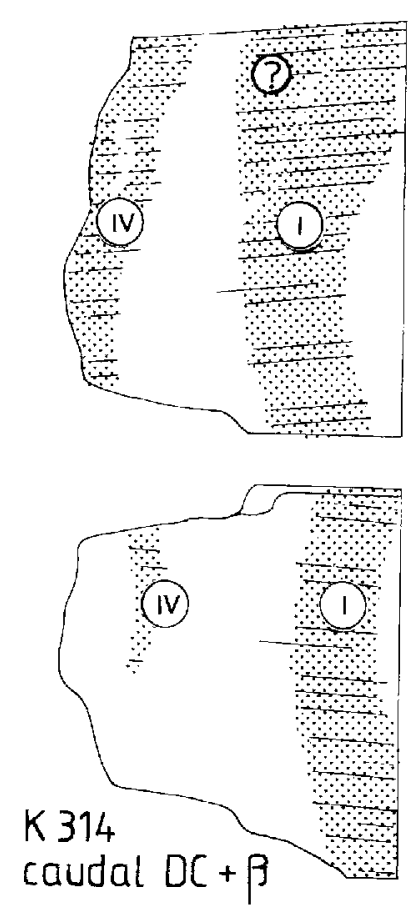

(b)

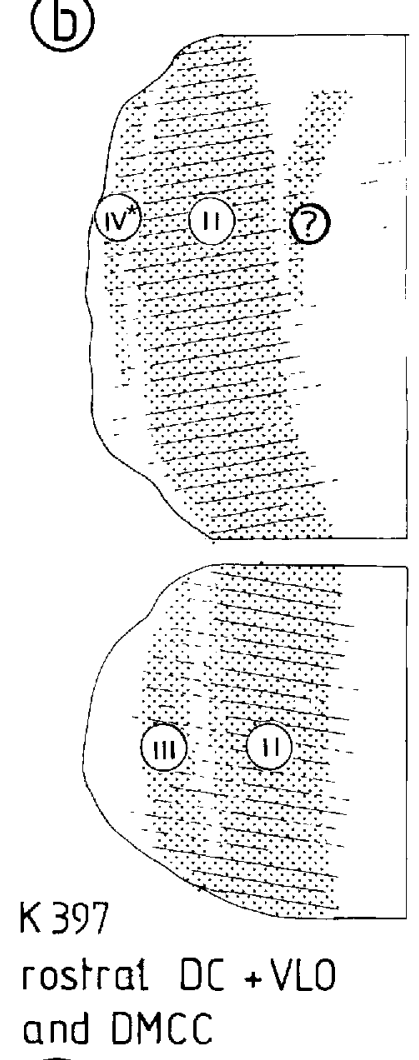

(e)

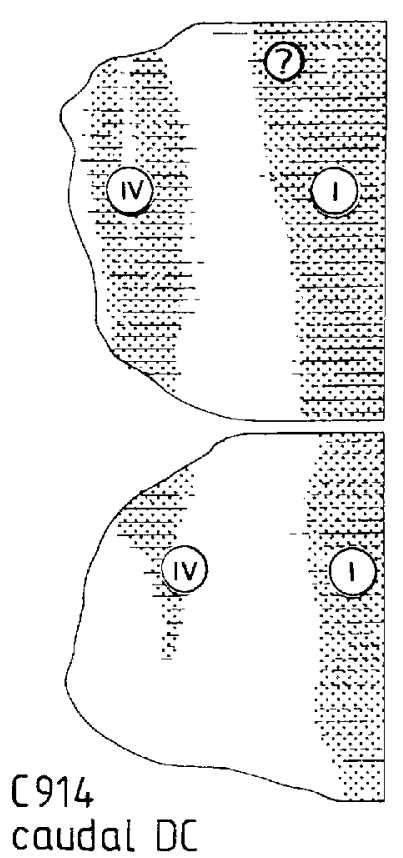

(C)
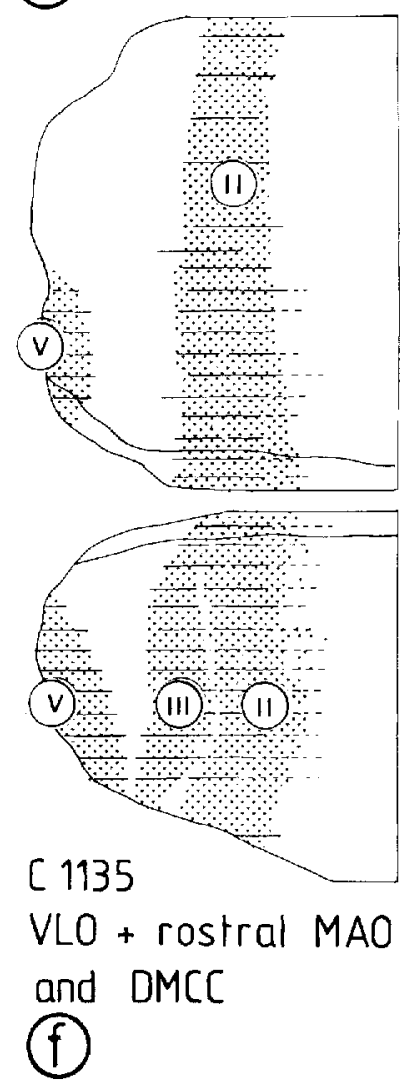

Fig. 7. a-f: Maps of the climbing fiber distribution on the ventral and dorsal surface of the nodulus. The presence of labeled climbing fibers in the sections used to prepare the maps is indicated by lines. Estimates of the climbing fiber zones are numbered I-V and indicated by dots. The intermediate, rostral dorsal cap/ventrolateral outgrowth (dc/ $/ \mathrm{vlo}$ ) innervated strip in $\mathrm{XZ}_{\mathrm{\Gamma V}}$ is indicated as $\mathrm{XZ}_{\mathrm{\Gamma V}}$. The ventromedial zone indicated with a question mark was labeled after injections in several parts of the inferior olive, including subnucleus $\beta$, the caudal and rostral dorsal cap (d,e), and in two of the cases involving the ventrolateral outgrowth (e). 


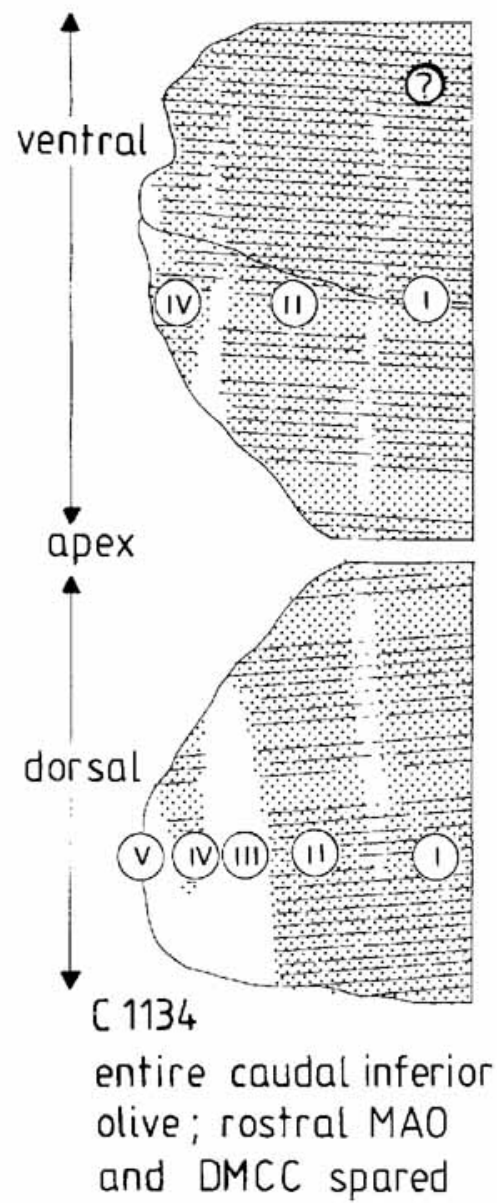

(a)
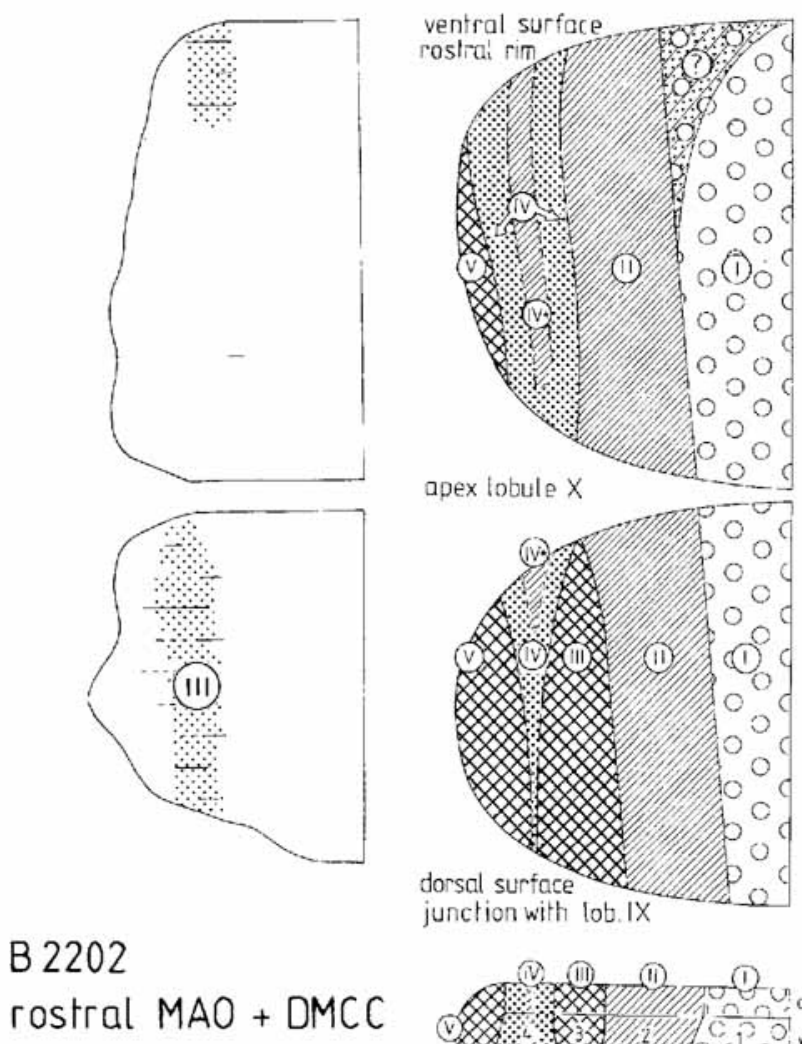

$1 \mathrm{~mm}$

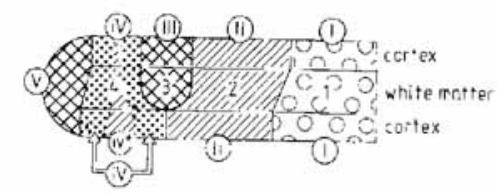

(c)
Fig. 8. a-c: Maps of climbing fiber distribution on the ventral and dorsal surface of lobule X. Same conventions as Figure 7. A summarizing diagram of the compartments and the climbing fiber zones of the nodulus is shown in $\mathrm{c}$. The white matter compartment corresponding to $\mathrm{XZ}_{V}$ cannot be distinguished in AChE-stained sections and is not indicated in $\mathrm{c}$.
Retrograde labeling of cells in the cerebellar nuclei does not occur with injections of tritiated leucine in the inferior olive. No labeled fibers were observed in the position of the nucleo-olivary tract (see also Fig. 19), which supports the interpretation that the labeling seen in this position with WGA-HRP injections of the inferior olive is retrograde.

\section{Projection of the rostral pole of the medial accessory olive (MAO) and the dorsomedial cell column (dmce)}

The two cases presented in this section display complementary labeling patterns. In one case a small injection of WGA-HRP was confined to the rostromedial part of the $\mathrm{MAO}$, the dmcc, and the tip of the ventral leaf of the PO (B 2202, Fig. 17D). This injection caused labeling of the $\mathrm{C}_{2}$ zone in the flocculus (Fig. 17C). In another rabbit (C 1134) large parts of the inferior olive, including the caudal dc, the rostral de, and the vlo, were injected with tritiated leucine, but the rostral pole of the MAO and the dmcc and the area of the $\mathrm{C}_{2}$ zone in the flocculus were spared (asterisk in Fig. 17B).

Labeled climbing fibers in case B2202 (Fig. 18) travel through the ventromedial part of the restiform body to enter the cerebellum rather caudally. They curve medially toward the midline over the anterior interposed nucleus and accumulate in the posterior interposed nucleus. From this fiber bundle, labeled fibers detach to enter the stalk of the paraflocculus. They terminate in a distinct $\mathrm{C}_{2}$ zone extending over the dorsal and the ventral paraflocculus and the caudal part of the flocculus (Fig. 18b-h). In the white matter of the flocculus and the paraflocculus these fibers occupy a region corresponding to the $\mathrm{C}_{2}$ compartment. Other labeled olivocerebellar fibers enter the caudal vermis, where they terminate in dorsal lobule $X$ and ventral lobule IX in a position corresponding to the $\mathrm{XZ}_{\mathrm{III}}$ zone (Figs. $8 \mathrm{~b}$, $18 \mathrm{f}-\mathrm{g}$ ). Retrogradely labeled cells are found in the posterior interposed nucleus. A few labeled neurons are present in the lateral cerebellar nucleus and its parvicellular extension. Some retrogradely labeled fibers were observed in the nucleo-olivary tract in the lateral angle of the fourth ventricle (Fig. 18a-c).

In case $C 1134$ the injection with tritiated leucine involved the caudal dc, rostral dc, and vlo (Fig. 17A). It extended into the caudal half of the MAO and DAO and subnucleus $\beta$, but spared the rostral pole of the MAO, 

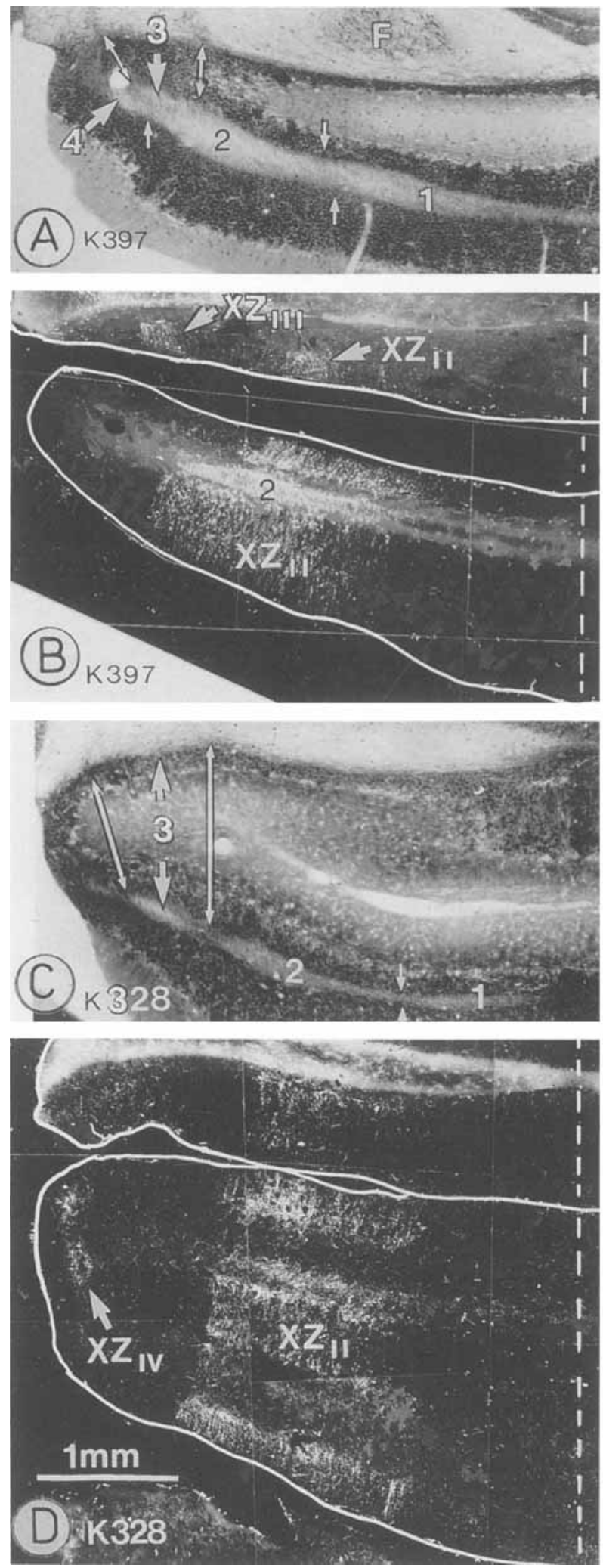

Fig. 9. Photographs of AChE-stained sections and darkfield exposures of sections containing labeled fibers in lobules IX and X. A, B, E: Case K 397. A.B: AChE. The raphes bordering compartment 3, which is present in dorsal lobule $\mathrm{X}$ and occupies the lateral white matter of lobule IX, and the raphe between compartments $\mathrm{XC}_{1}$ and $\mathrm{XC}_{2}$ are indicated with small white arrows in $A$ and $E$. The compartments are indicated with arabic numerals. WGA-HRP-labeled fibers from the rostral dc and the vlo are present in $\mathrm{XZ}_{\mathrm{II}}$ and compartment $\mathrm{XC}_{2}$. Labeled climbing fibers from the dmcc are present in compartment $\mathrm{XC}_{3}$ and
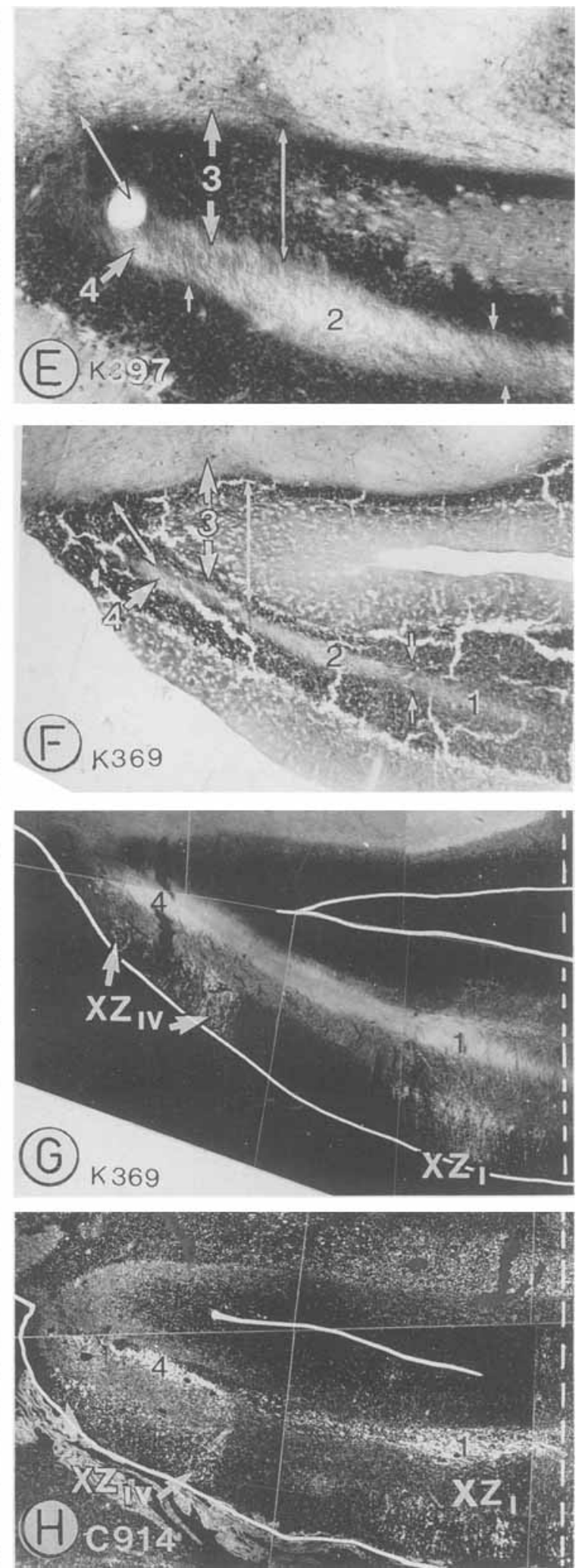

terminate laterally in Iobule IX farrows in B). C, D: Case K 328 . WGA-HRP-labeled fibers from the rostral de and vlo are present in compartments $\mathrm{XC}_{2}$ and $\mathrm{XC}_{4}$ and in the corresponding zones. $\mathbf{F}, \mathbf{G}$ : Case $\mathrm{K} 369$. WGA-HRP-labeled fibers after an injection in the caudal dc and subnucleus $\beta$ are present in $\mathrm{XC}_{1}$ and $\mathrm{XC}_{4}$. $\mathrm{H}$ : Case $\mathrm{C}$ 914, with an injection of $\left[{ }^{3} \mathrm{H}\right.$ leucine in the caudal de, shows a distribution similar to that of $\mathrm{K} 369$. HRP-reactive erythrocytes were retouched in montages $B, D$, and $G$. The midline in $B, D, G$, and $H$ is indicated by a dashed line. 


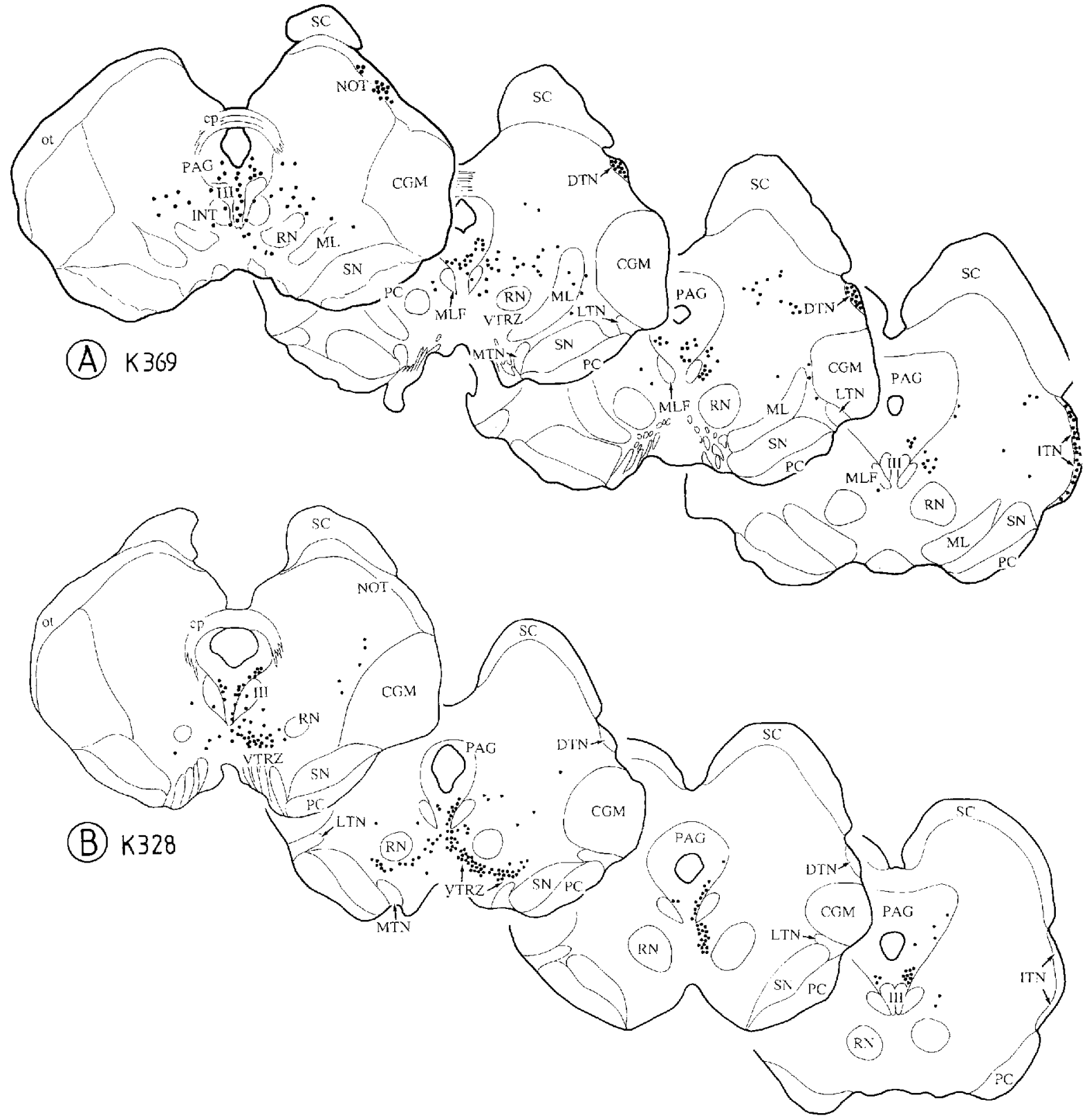

Fig. 10. Drawings of transverse sections through the mesencephalon in case $K 369$ (A) with an injection involving the cde and in case $K$ 328 (B) with an injection involving the rdc/vlo. Black dots indicate retrogradely labeled cells. The VTRZ is labeled in case $\mathrm{K} 328$; the nucleus of the optic tract (NOT) and the dorsal and interstitial terminal nuclei of the accessory optic system (DTN, ITN) are labeled in case $\mathrm{K} 369$. which contains the neurons projecting to the $\mathrm{C}_{2}$ zone. Dense labeling was present over all parts of the white matter and the molecular layer of the flocculus except for a small caudal area (asterisk in Fig. 17A, Fig. 19B) that corresponds to the lateral, unlabeled area seen in the caudal flocculus in rostral de/vlo injected cases and the labeled $\mathrm{C}_{2}$ compartment in case B 2202. The white matter of case C 1134 was characterized by relatively sparse labeling at the borders between the compartments. These areas of sparse labeling probably coincide with the AChE-positive raphes (Fig. 19A). Labeling in the nodulus spared the $\mathrm{XC}_{3}$ compartment and the $\mathrm{XZ}_{\mathrm{III}}$ zone, as well as the molecular layer covering the lateral pole of the lobule, corresponding to $X Z_{\mathrm{V}}$
(Figs. 8a, 16B). Labeled olivocerebellar fibers were also found in the fastigial nucleus, the medial part of the vermis and paramedian lobule, the dorsal part of the simple lobule, and in several small bands in the ventral paraflocculus and the ventral part of the dorsal paraflocculus. This large injection of the anterograde tracer $\left[{ }^{3} \mathrm{H}\right]$ leucine caused no labeling in the nucleo-olivary tract (asterisk in Fig. 19).

\section{Conclusions}

The olivocerebellar projection to the flocculus and the nodulus is summarized in the diagrams of Figures $8 \mathrm{c}$ and 20. 


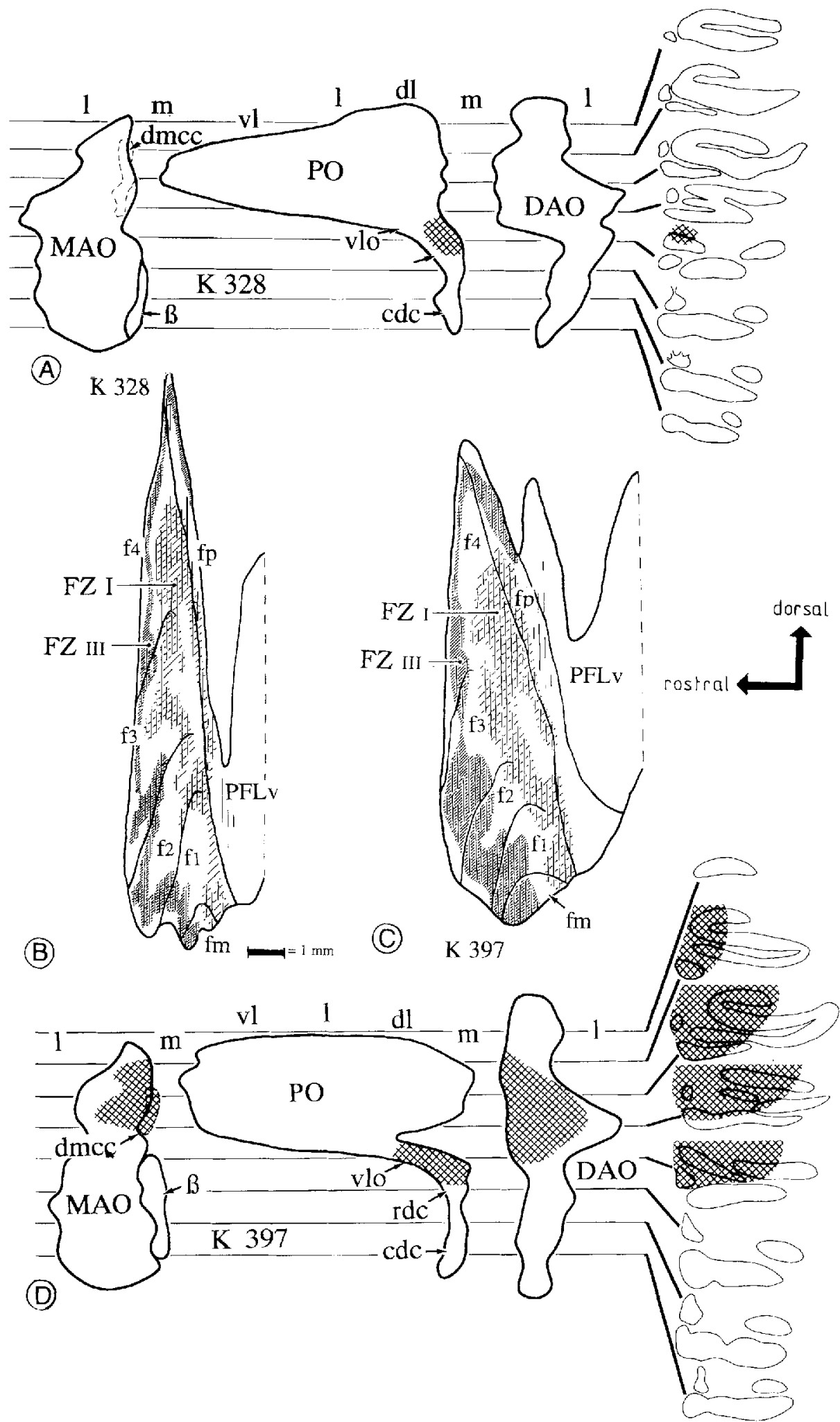

Fig. 11. Diagrams of the distribution of climbing fibers in the unfolded flocculus and ventral parafiocculus and diagrams of the inferior olive showing injection sites including the rostral dorsal cap and ventrolateral outgrowth, in cases K 328 (A, B) and K 397 (C, D). Labeled climbing fibers in sections used to prepare the unfolded flocculus are indicated with vertical lines in $\mathrm{B}$ and $\mathrm{C}$. The extent of zones $\mathrm{FZ}_{\mathrm{I}}$ and $\mathrm{FZ}_{\mathrm{III}}$ is indicated with coarse hatching and with fine hatching, respectively. Scale in $\mathrm{B}$ also applies to $\mathrm{C}$, and the rostrocaudal scale is the same as the dorsoventral scale. 


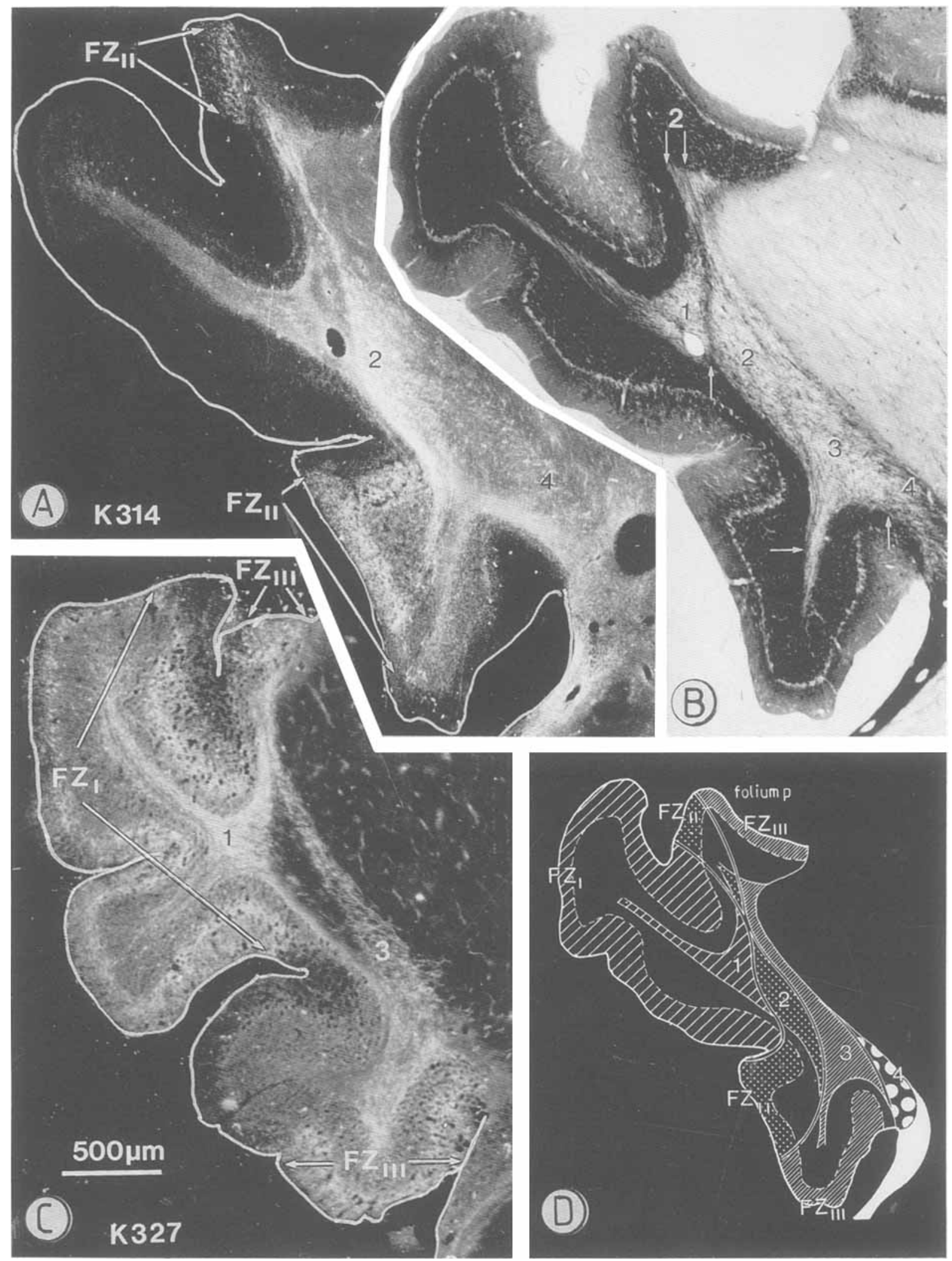

Fig. 12. Darkfield photographs of tetramethylbenzidine (TMB)processed sections through the flocculus in $\mathrm{K} 314$ (A) with an injection in the caudal dorsal cap and $\mathrm{K} 327$ (C) with an injection in the rostral dorsal cap. Raphes and white matter compartments are depicted in adjacent AChE-stained section from $\mathrm{K} 314$ (B) and corresponding diagram (D). Note that the labeled parts of the white matter and associated floceular cortex in $\mathrm{K} 314\left(\mathrm{FC}_{2}, \mathrm{FC}_{4}\right.$, and $\left.\mathrm{FZ}_{\mathrm{II}}\right)$ are not labeled in $\mathrm{K} 327$, which shows prominent labeling in $\mathrm{FC}_{1}, \mathrm{FC}_{3}, \mathrm{FZ}_{\mathrm{I}}$, and $\mathrm{FZ}_{\mathrm{II}}$. 


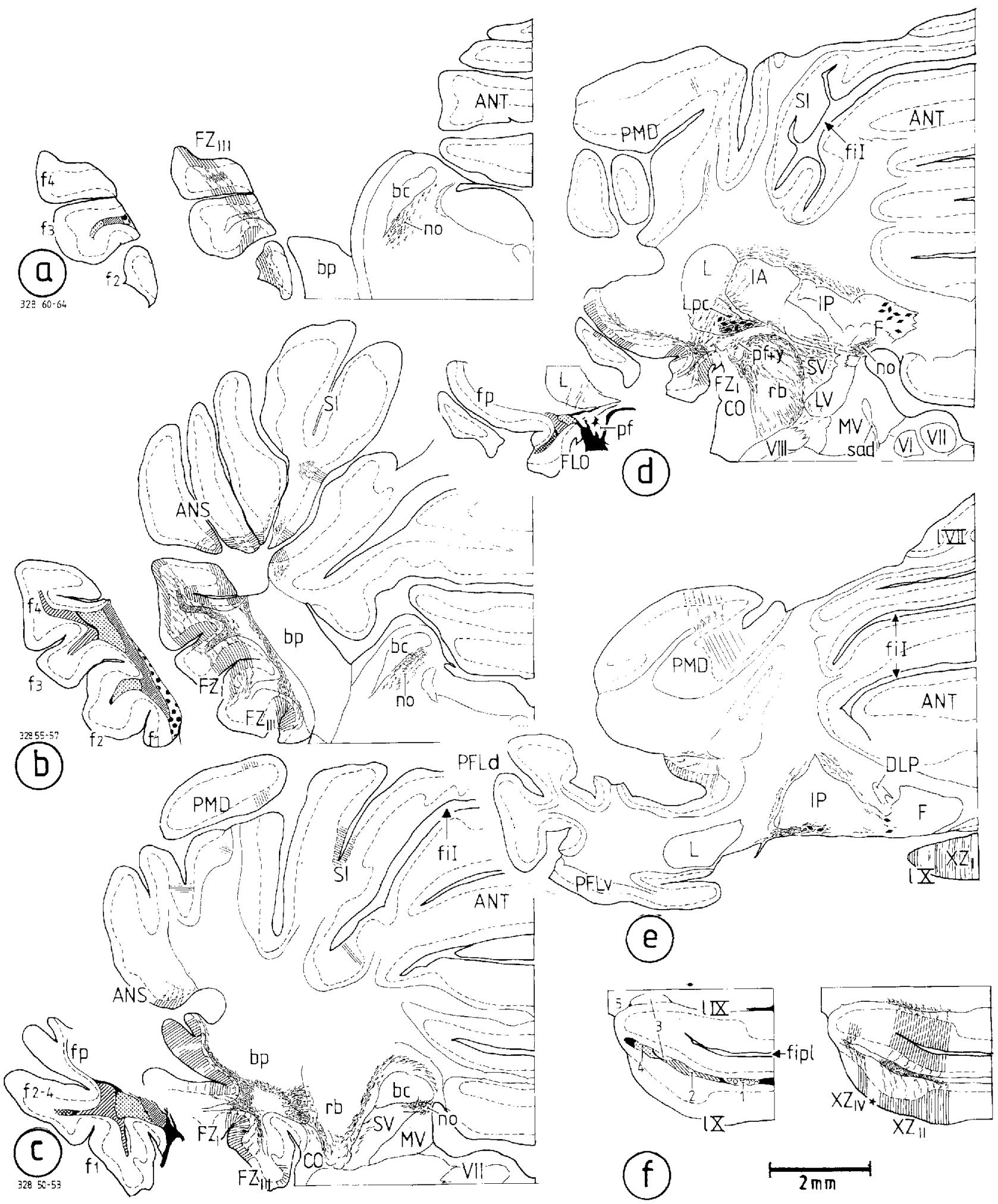

Fig. 13. a-f: Drawings of adjacent AChE- and HRP-reacted transverse sections through brainstem and cerebellum in case $\mathrm{K} 328$. Note the presence of labeled fibers in $\mathrm{FC}_{1}$ and $\mathrm{FC}_{3}$ and in $\mathrm{FZ}_{1}$ and $\mathrm{FZ}_{I I}$ in the

fiocculus and in compartments and zones 1 and 4 of lobule X. For symbols indicating compartments in AChE-stained sections see Figure 4. 


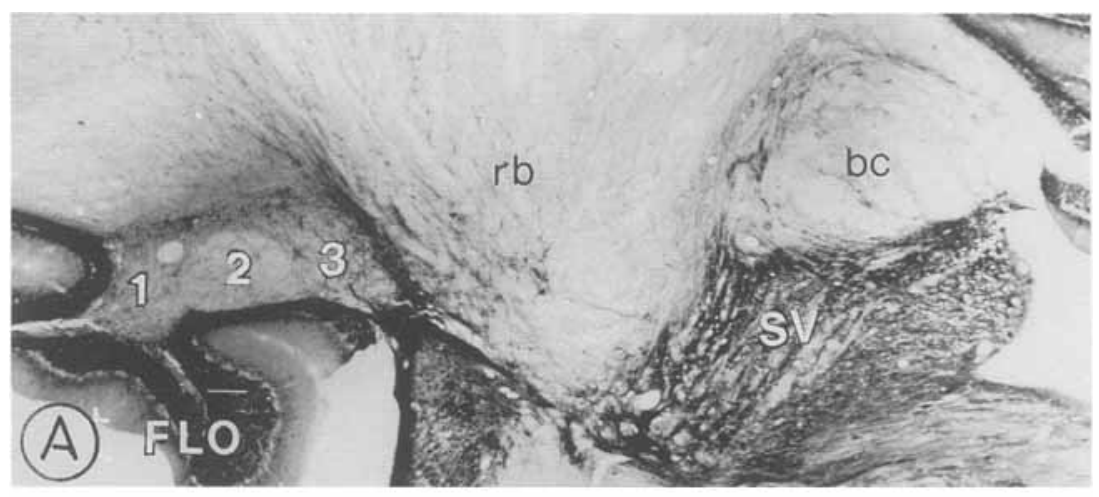

J. TAN ET AL.
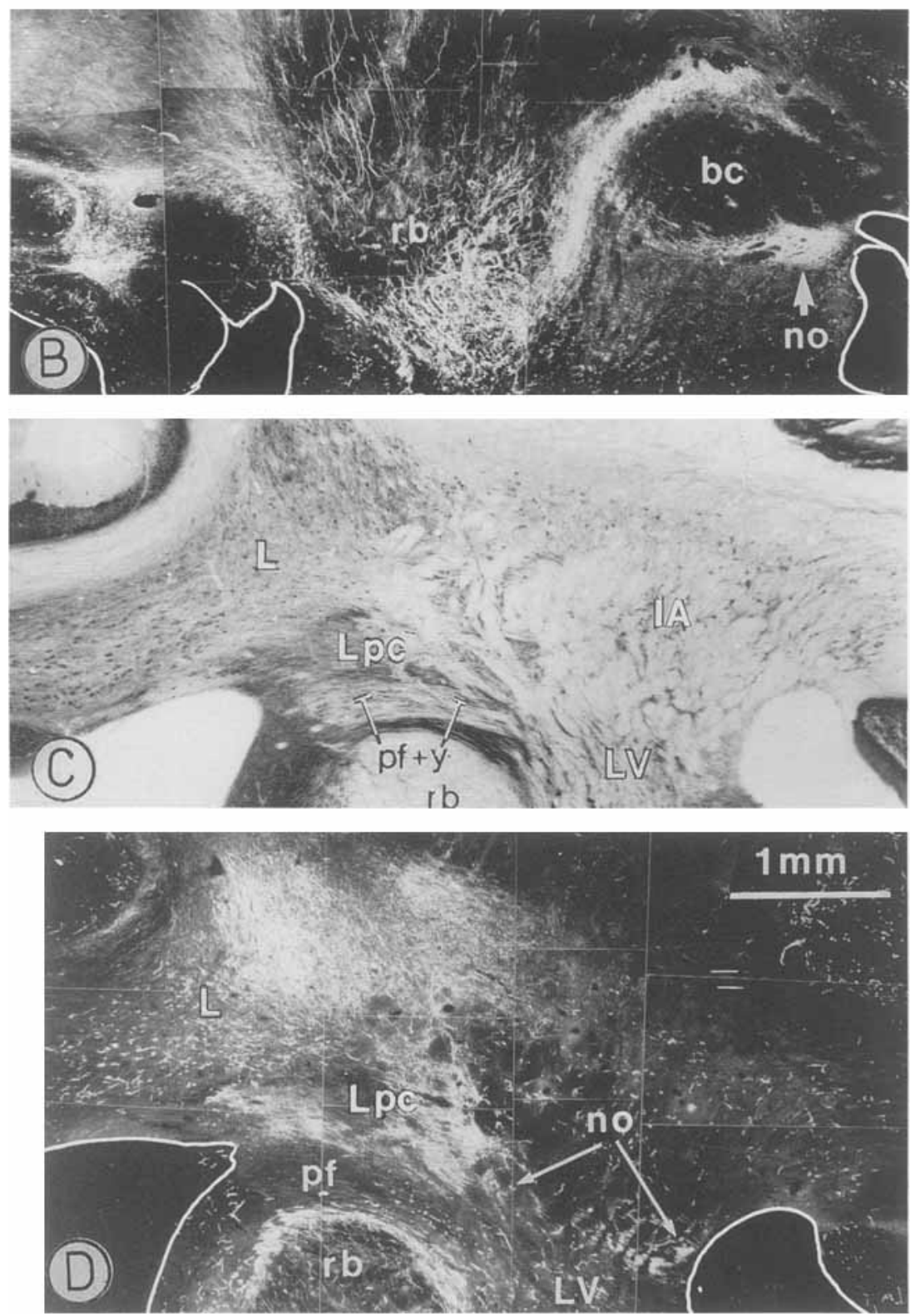

Fig. 14. Brightfield photographs of AChE-stained sections (A, C) and darkfield photographs of WGA-HRP-reacted transverse sections (B, D) through brainstem and flocculus in K 397. A, B: Anterogrado labeling is present in compartments $\mathrm{FC}_{1}$ and $\mathrm{FC}_{3}$ and retrograde labeling in axons of the nucleo-olivary pathway (no) and in neurons of the cerebellar nuclei. C, D: Fibers of the nucleo-olivary pathway (no) collect at this more caudal level near the lateral angle of the fourth ventricle. The floccular peduncle (pf) does not contain labeled fibers. 


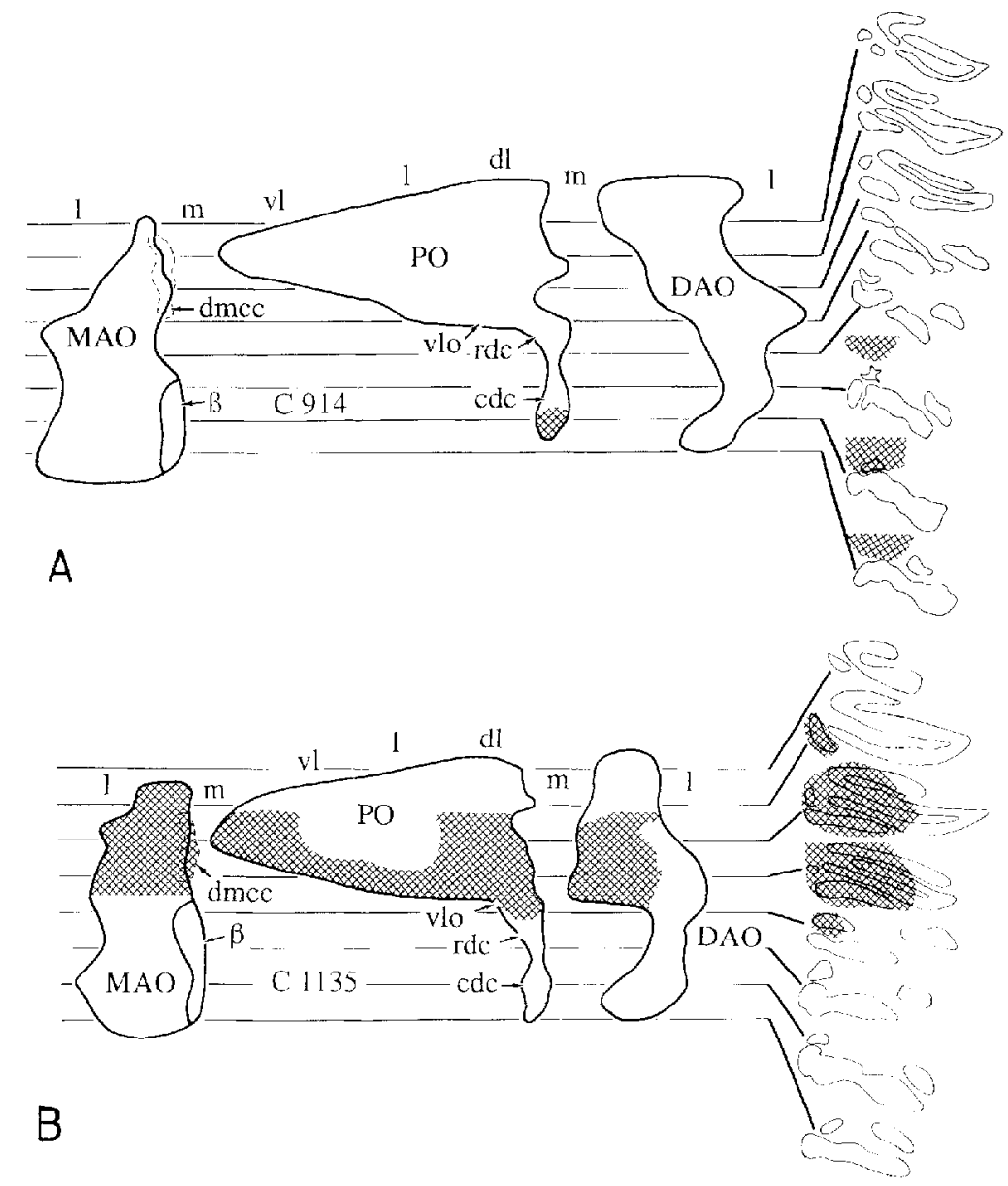

Fig. 15. A, B: Diagrams of the injection sites in the inferior olive of cases C 914 and C 1135.

1. Labeled olivocerebellar fibers in the flocculus from injections including the caudal dc (i.e., the region caudal to the constricted portion of this subnucleus - cases K 369 , $\mathrm{K} \mathrm{314,} \mathrm{K} 426$, and $\mathrm{C} 914$ ) become located in compartments $\mathrm{FC}_{2}$ and $\mathrm{FC}_{4}$ of the white matter of the flocculus, which are delineated by $\mathrm{AChE}$-positive raphes in adjacent AChE-stained sections. They terminate in the corresponding cortical zones $\mathrm{FZ}_{\mathrm{II}}$ and $\mathrm{FZ}_{\mathrm{IV}}$ (Fig. 20). Injections involving the rostral $\mathrm{dc}$ and vlo result in labeling of fibers in compartment $\mathrm{FC}_{1}$ and $\mathrm{FC}_{3}$, which terminate in $\mathrm{FZ}_{\mathrm{I}}$ and $\mathrm{FZ}_{\mathrm{III}}$. These zones extend into folium $\mathrm{p}$ and merge around the rostral tip of $\mathrm{FZ}_{\mathrm{II}}$ (Fig. 20). Some labeling in compartment $\mathrm{FC}_{1}$ and zone $\mathrm{FZ}_{\mathrm{I}}$ is observed in $\mathrm{K}$ 314, where the caudal dc injection site encroaches on the most caudal part of the rostral dc. The $\mathrm{C}_{2}$ compartment is located laterally and caudally in the white matter of the flocculus. It is continuous with a compartment in the medial white matter of the paraflocculus and contains labeled fibers that terminate in the corresponding $\mathrm{C}_{2}$ zone after injections including the rostral pole of the MAO (C 1135 and B 2202).

2. In the white matter of the nodulus four compartments $\left(\mathrm{XC}_{1-4}\right)$ are delineated by accurnulations of $\mathrm{AChE}$ at their borders. Injections in the caudal dc and subnucleus $\beta$ result in labeled fibers in compartments $X_{1}$ and $X_{4}$ that terminate as climbing fibers in the $X Z_{I}$ and $X Z_{T V}$ zones (Fig. 8c). Zone $\mathrm{XZ}_{\mathrm{IV}}$ is bisected by a central, empty strip of molecular layer and is wider on the ventral than on the dorsal side of lobule X. Injections in the rostral $\mathrm{dc} /$ vlo label zone $\mathrm{XZ}_{\text {II }}$ on the ventral and dorsal surface of lobule $\mathrm{X}$ and the central strip in $\mathrm{XZ}_{\mathrm{IV}}$ (indicated as $\mathrm{XZ}_{\mathrm{TV}^{*}}$. A rostrolateral area in $\mathrm{XZ}_{\mathrm{I}}$ contains labeled climbing fibers after injections of subnucleus $\beta$, the caudal dc, and the rostral dc and vlo. This area is indicated with a question mark in Figures 7 and 8 . Compartment $\mathrm{XC}_{3}$ is wedged between compartments $\mathrm{XC}_{2}$ and $\mathrm{XC}_{4}$ and terminates only in the dorsal cortex of the lobule. It is continuous with a distinct, much wider compartment in the lateral white matter of lobule IX. Labeling in $\mathrm{XZ}_{\mathrm{III}}$ and $\mathrm{XZ} \mathrm{V}_{\mathrm{y}}$ is in accordance with the data from the retrograde labeling experiments of Katayama and Nisimaru (1988). $\mathrm{XC}_{3}$ and $\mathrm{XZ}_{\mathrm{II}}$ are labeled when the injection includes the dmcc (K 397, B 2202, and C 1135). Labeling is present in a lateral strip of climbing fibers covering the lateral rim of the nodulus $\left(\mathrm{XZ}_{\mathrm{V}}\right)$ when the injection of the rostral MAO includes its lateral border region (C 1135), but not when this region is spared (C 1134 and B 2202). 


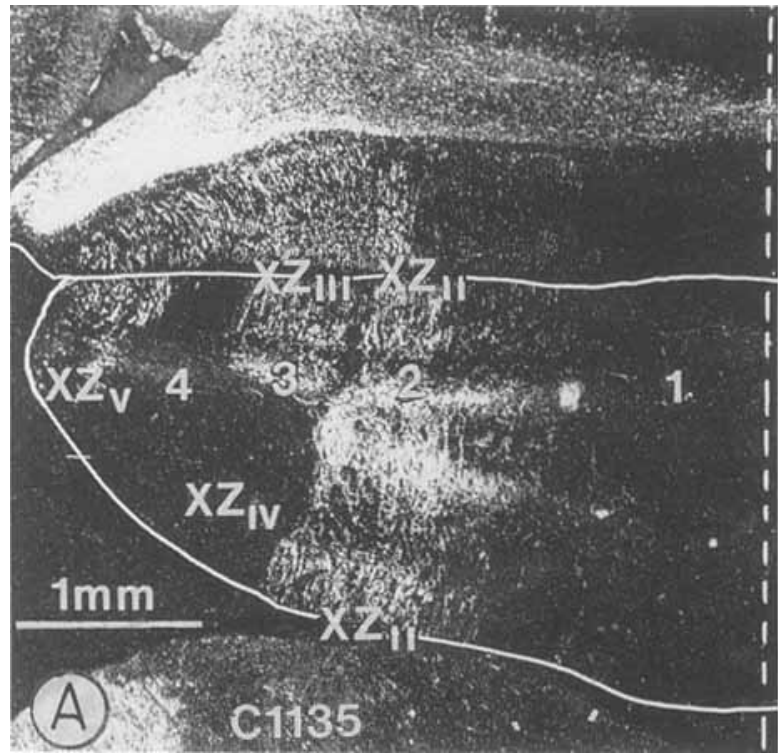

Fig. 16. Darkfield photographs of autoradiograms of sections through lobules IX and X. A: Case C 1135. This injection involved the vlo and the rostral MAO and labeled fibers in compartments $\mathrm{XC}_{2}$ and $\mathrm{XC}_{3}$ and the corresponding zones and in the extreme lateral pole of

\section{DISCUSSION}

\section{Correlation of white matter compartments with climbing fiber zones in the flocculus}

The present study showed that fibers from certain subnuclei of the inferior olive segregate into bundles that occupy specific compartments within the white matter of the flocculus and the nodulus. They travel within these compartments until they penetrate the granular layer to terminate in the molecular layer as climbing fibers on their target Purkinje cells. Climbing fibers projecting through these compartments constitute zones that are continuous across the folia of the flocculus and the nodulus and sometimes extend into adjacent lobules. The graphical reconstructions of the climbing fiber zones of the flocculus (Figs. 2, 11, 17) exemplify the approximately orthogonal relation between the zones and the transverse interfolial fissures. The AChE-positive raphes, which subdivide the floccular white matter into compartments (Tan et al., 1995), shift medially in the direction of the caudal pole of the flocculus. A similar shift was observed in the position of the climbing fiber zones $\mathrm{FZ}_{\mathrm{I}-\mathrm{IV}}$.

The caudal dc, the rostral dc, and the vlo, first described by Kooy (1916), are segments of a continuous cell column located dorsomedially to the MAO. The borders between these segments were never precisely defined with anatomical methods, but they have been identified in the rabbit by recording the presence or absence of field potentials in the $\mathrm{dc}$ and vlo after flash stimulation of the ipsilateral and the contralateral eyes (Maekawa and Takeda, 1977; Takeda and Maekawa, 1980) and on the basis of the laterality and the orientation of the best-response axis for rotation of the visual surround (Simpson et al., 1981; Leonard et al., 1988). The border between the rostral and caudal dc was found to be located at the constriction of the cell column, which marked the transition between units in the caudal dc that were best activated from the contralateral eye by temporal

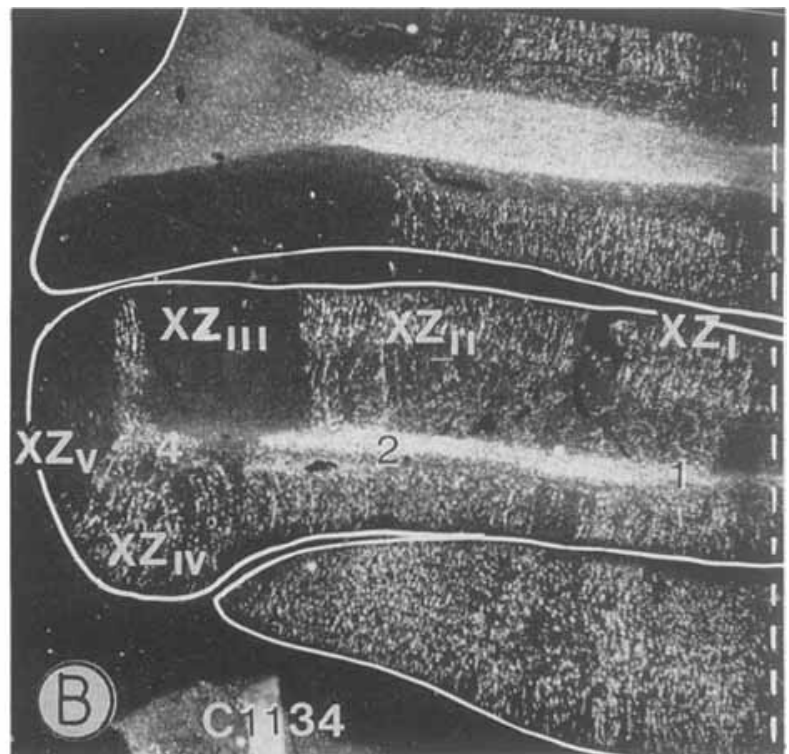

lobule $\mathrm{X}$ (zone $\mathrm{XZ} Z_{\mathrm{V}}$ ). B: Case $\mathrm{C} 1134$. This large injection spared the rostral MAO and the dmcc. No labeling is observed in compartments $\mathrm{XC}_{3}$ and zones $\mathrm{XZ}_{\mathrm{III}}$ and $\mathrm{XZ}_{\mathrm{V}}$.

to nasal rotation around the vertical axis (vertical axis neurons) and the more rostral region comprising the rostral dc and vlo, containing units that were best activated by rotation around a horizontal axis that is approximately co-linear with the axis perpendicular to the contralateral anterior semicircular canal. Stimulation of the ipsilateral eye dominates the response of most cells in the rostral dc [anterior $\left(45^{\circ}\right)$ axis neuronsl. Cells in the vlo and in the most rostral part of the rostral de prefer stimulation of the contralateral eye [posterior $\left(135^{\circ}\right)$ axis neurons|. The border between the caudal dc and the rostral de is in accordance with the general pattern of afferent connections of the caudal dc from the NOT, the DTN, and the ITN, and of the rostral de and the vlo from the MTN and the VTRZ.

Several previous anatomical studies found a zonal arrangement in the climbing fiber projection to the flocculus in various species (Yamamoto, 1979a; Gerrits and Voogd, 1982; Sato et al., 1983; Ruigrok et al., 1992). Injections of HRP in the rabbit flocculus resulted in different patterns of retrograde labeling in the olive (Yamamoto, 1979a). A few small injections in the rostral and middle portions of the flocculus resulted in retrograde labeling mainly restricted to the rostral dc. Most injections that covered folia f1-4 showed labeled cells in the rostral dc with the vlo and in the caudal dc, separated by an empty segment of the dc. This empty segment of the dc and the principal olive contained labeled cells when the injection included folium $p$.

Similar observations were made in the combined retrograde WGA-HRP and anterograde Phaseolus vulgaris lectin tracing study of the olivocerebellar projection in the rat (Ruigrok et al., 1992). 'The caudal de was found to project to paired zones, $\mathrm{FE}$ and $\mathrm{FE}^{\prime}$, in the flocculus, whereas a more rostral segment of the dc, corresponding to the "empty segment" of Yamamoto (1979a), projected to the extension of the FE zone into the ventral paraflocculus. The vlo innervated two other floccular zones (FD and FD'), which interdigitated with and were situated caudal to $\mathrm{FE}$ and $\mathrm{FE}^{\prime}$, 

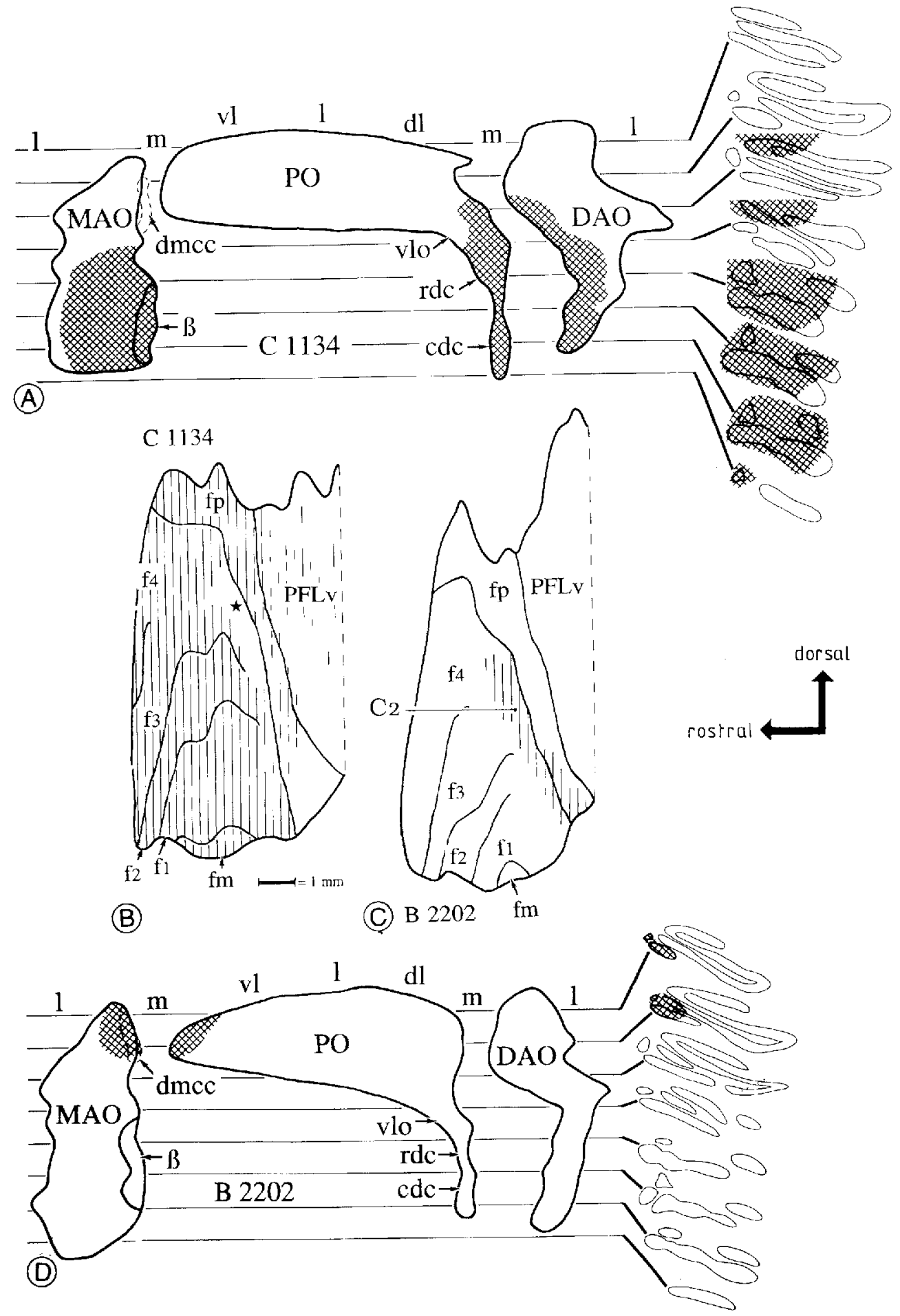

Fig. 17. Diagrams of the distribution of climbing fibers in the unfolded flocculus and ventral paraflocculus and diagrams of the inferior olive showing injection sites in case C. $1134(\mathbf{A}, \mathbf{B})$ with an injection including cde, rdc, and vlo, but sparing the rostral MAO and the dmce, and for case B $2202(C, D)$ with an injection in rostral MAO.

respectively. More rostrally located cells in the $\mathrm{PO}$ projected to an extension of the FD and FD' zones in the ventral paraflocculus. FD and $\mathrm{FD}^{\prime}$ fused around the end of the FE
Note that in C 1134 almost all parts of the floceulus are labeled except for a caudolateral region corresponding to the $C_{2}$ zone (asterisk), which does receive climbing fibers in case $\mathrm{B} 2202$. Scale in $\mathrm{B}$ applies also to $\mathrm{C}$, and the rostrocaudal scale is the same as the dorsoventral scale.

zone and continued as the $\mathrm{D}$ zone in the ventral paraflocculus. Both Yamamoto (1979a) and Ruigrok et al. (1992) recognized the projection of the rostral MAO to the caudal 


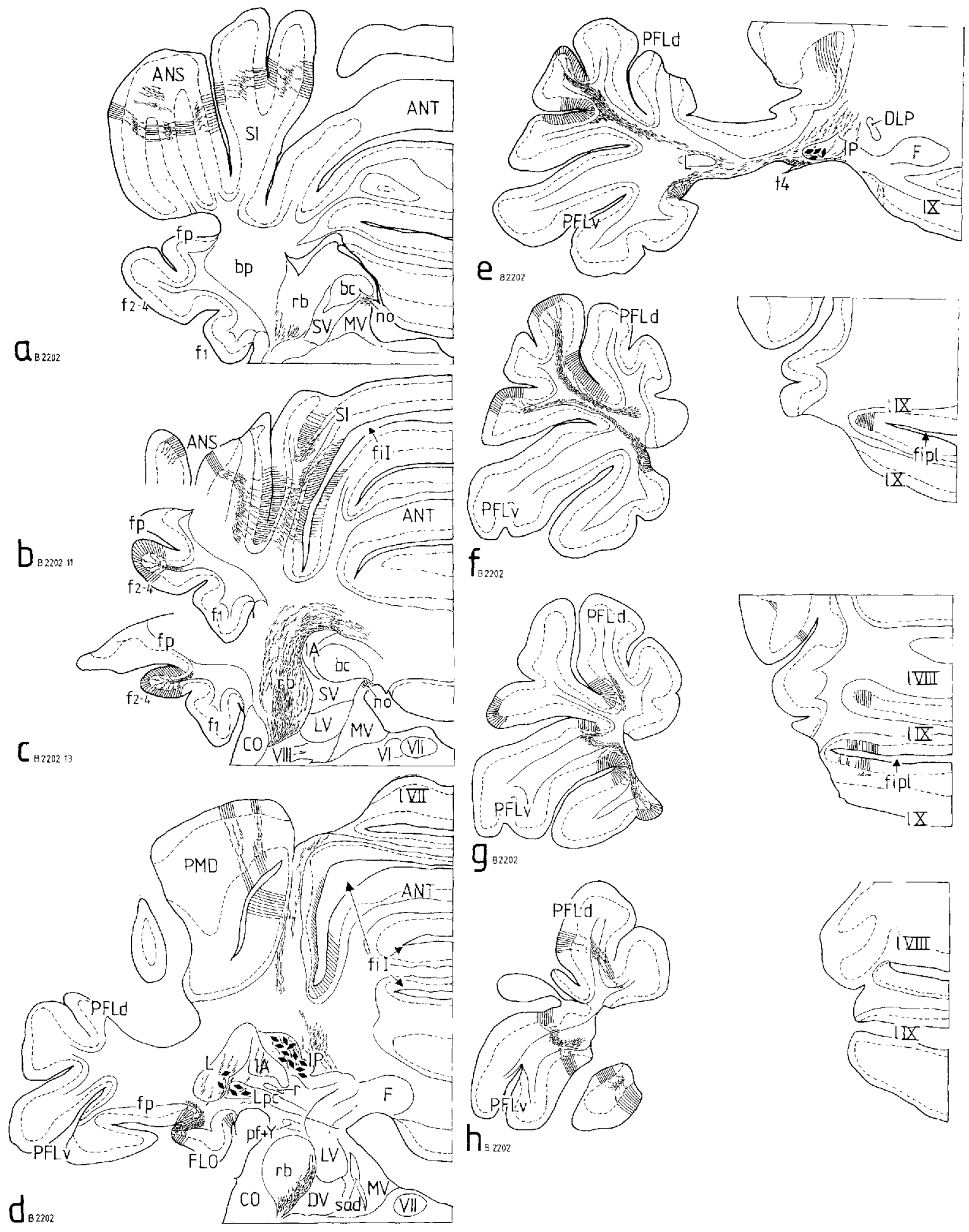

Fig. 18. a-h: Drawings of labeled fibers in transverse sections through the brainstem and cerebellum in case $B$ 2202. Labeling is present in the $C_{2}$ compartment and zone and in a region corresponding to compartment $\mathrm{XC}_{3}$ in lobules IX and $\mathrm{X}$.

flocculus, which continues in the ventral paraflocculus as the $\mathrm{C}_{2}$ zone. Yamamoto's (1979a) observations also indicated that cells projecting to more rostral segments of the zones $\mathrm{FZ}_{\mathrm{I}-\mathrm{IV}}$ are located more rostrally in the caudal dc and in the rostral dc/vlo column. Such a shift was not observed with the relatively large injections in the $\mathrm{dc} / \mathrm{v} \mathrm{l}_{\mathrm{o}}$ in our experiments.

A more intricate pattern in the climbing fiber projection to the flocculus has been shown by Gerrits and Voogd (1982) in the cat. Using an anterograde autoradiographic 

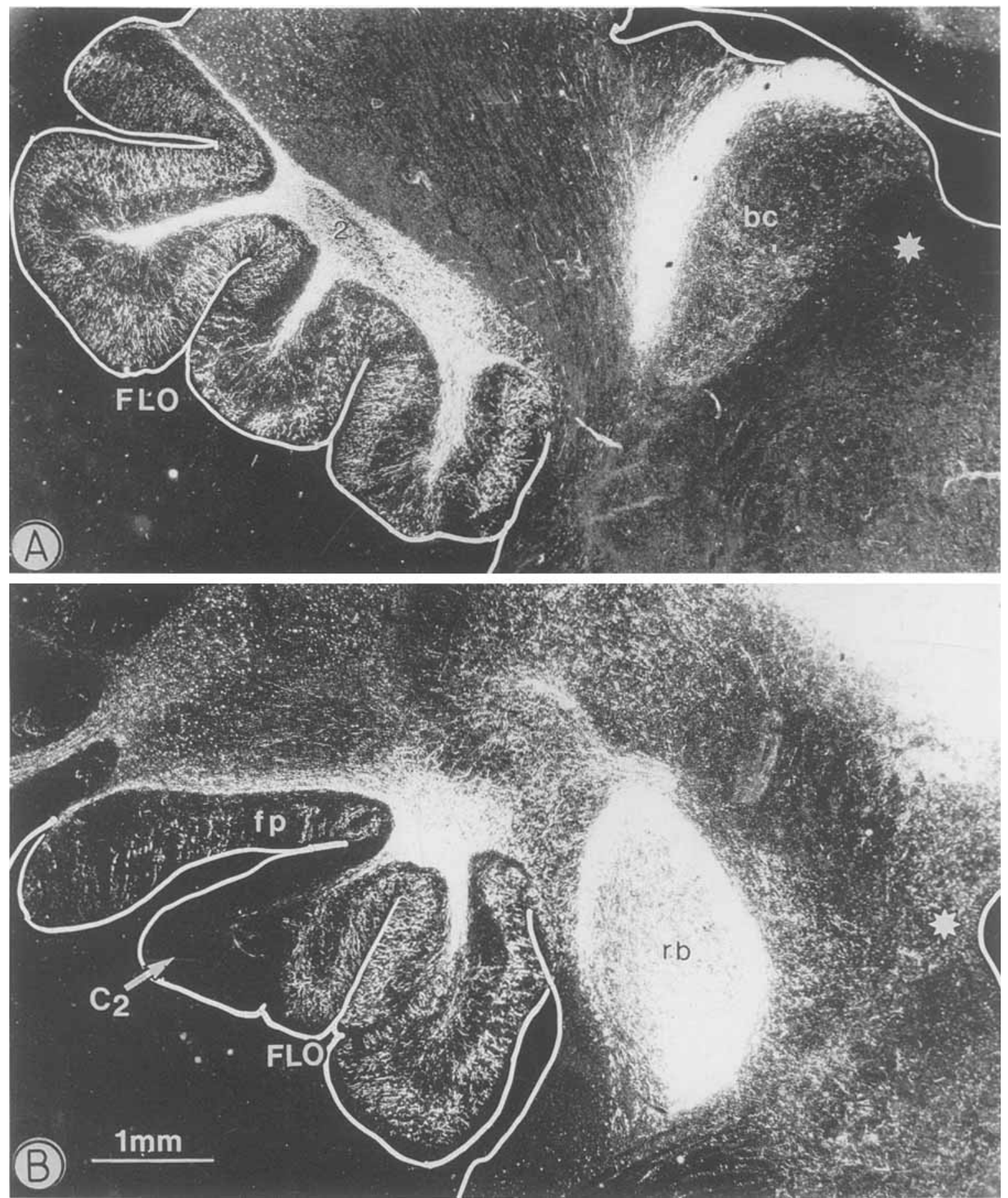

Fig. 19. Darkfield photomicrographs of transverse sections through the flocculus in case C 1134. A: Note the relatively sparse number of labeled fibers at the borders of the white matter compartments $\mathrm{FC}_{\mathrm{I}-3}$ and the dense labeling in the lateral part of compartment $\mathrm{FC}_{2}$. B: In the caudal flocculus labeled fibers are absent from compartment $\mathrm{C}_{2}$. Also, no labeling is present in the position of the nucleo-olivary tract (star in A and B; compare with Fig. 14B,D). technique, they found that the caudal dc, rostral dc, and vlo each supplied two zones of Purkinje cells in the flocculus. Their medial $F 1$ zone received a projection from the caudal $\mathrm{dc}$ and did not extend into the paraflocculus. It was bordered on its lateral side by the $\mathrm{F} 2$ zone, which received fibers from the vlo. The $\mathrm{F} 1 / \mathrm{F} 2$ border is considered genuine because it separates climbing fibers from noncontiguous portions of the inferior olive. The F1 zone of the cat, therefore, can be considered as the equivalent of the $\mathrm{FZ}_{\mathrm{TV}}$ zone in the rabbit and the $\mathrm{FE}^{\prime}$ zone in the rat. The vlo-innervated $\mathrm{F} 2$ zone in the cat is bordered on its lateral side by $\mathrm{F} 3$, which receives a projection from the rostral dc. The same pattern is repeated more laterally, with the appearance of F4 (caudal dc), $\mathrm{F} 5$ (vlo), and $\mathrm{F} 6$ (rostral dc). 

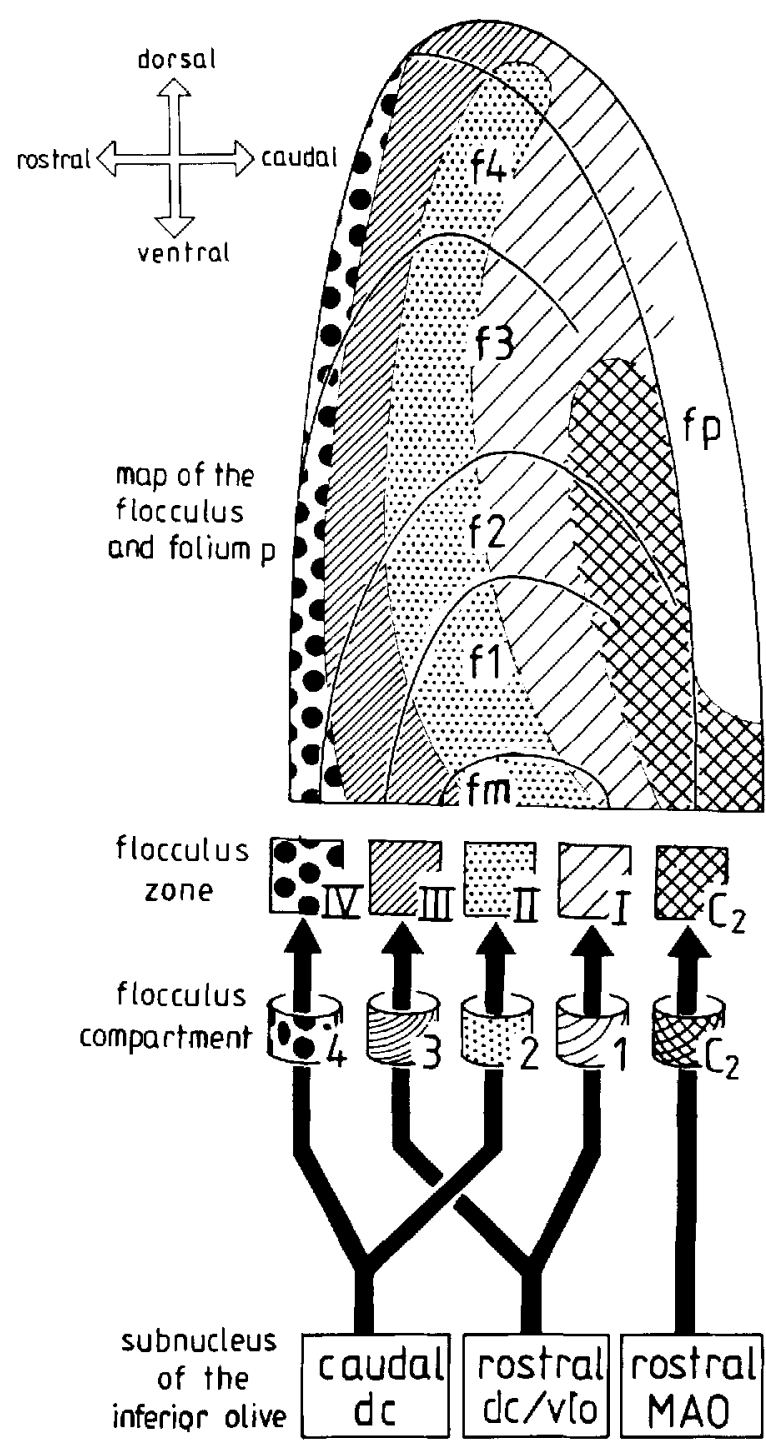

Fig. 20. Summary diagram of the projection of the de, vlo, and rostral MAO through compartments $\mathrm{FC}_{1-4}$ and $\mathrm{C}_{2}$ to the corresponding zones $\mathrm{F}_{\mathrm{I}}-\mathrm{F} Z_{\mathrm{IV}}$ and $\mathrm{C}_{2}$ in the flocculus.

The vlo and rostral dc zones continue across the posterolateral fissure into the adjoining medial extension of the ventral paraflocculus (ME), where they enclose the end of the caudal dc-innervated $\mathrm{F} 4$ zone together with the climbing fibers of the F7 zone, which take their origin from the caudal bend of the principal olive and which are present exclusively in the ME. This pattern is similar to the situation in the rabbit and the rat, and, consequently, F1 and $\mathrm{F} 4$ of the cat can be considered as the equivalents of our $\mathrm{FZ}_{\mathrm{IV}}$ and $\mathrm{FZ}_{\mathrm{II}}$ zones and the combined $\mathrm{F} 2 / 3$ and $\mathrm{F} 5 / 6$ zones of the cat as the homologues of the $F Z_{\mathrm{III}}$ and $F Z_{1}$ zones of the rabbit flocculus. A subdivision of the $\mathrm{FZ}_{\mathrm{I}}$ and $\mathrm{FZ}_{111}$ zones was not observed in our material, although an additional AChE-positive raphe that divided the $\mathrm{FC}_{1}$ compartment over some of its extent was sometimes present (Tan et al., 1995).

The studies of Yamamoto (1979a), Gerrits and Voogd (1982, 1989), and Ruigrok et al. (1992), as well as our own observations, lead to the conclusion that an identical pattern of climbing fiber connections is present in the flocculus of different mammalian species and that this pattern finds its expression in the compartmental subdivision of the white matter of the flocculus of the rabbit as revealed in AChE-processed brain sections (Tan et al., 1995). Sato et al. (1983) distinguished three zones in the flocculus of the cat, with projections from both the rostral de and the vlo to rostral and caudal zones, and projections from the caudal dc to a middle zone. They did not recognize the projection from the rostral MAO to the $\mathrm{C}_{2}$ zone of the flocculus nor a second zone receiving climbing fibers from the caudal dc. Their zones resemble $\mathrm{FZ}_{\mathrm{T}}, \mathrm{FZ}_{\mathrm{II}}$, and $\mathrm{FZ}_{\mathrm{III}}$ of the rabbit. A similar trizonal organization was also found in the flocculus of the monkey (Balaban et al., 1981).

\section{Correlation of white matter compartments with climbing fiber zones in the nodulus}

Our observations on the projection of the inferior olive to the nodulus confirm the description of Katayama and Nisimaru (1988), based on retrograde axonal transport of HRP, and resemble the pattern reported by Balaban and Henry (1988), also in the rabbit. We distinguished four compartments in AChE-stained material in each half of the white matter of the nodulus. A paramedian, 1-mm-wide $\mathrm{XZ}_{\mathrm{T}}$ zone was innervated through compartment $\mathrm{XC}_{1}$ by subnucleus $\beta$ and the caudal dc. The separate contributions of these two subnuclei could not be distinguished in our experiments. Katayama and Nisimaru (1988) subdivided this zone into a medial, $\beta$-innervated zone and a lateral, caudal dc-innervated zone. We distinguished a triangular rostrolateral area bordering on $\mathrm{XZ}_{\mathrm{I}}$ in the ventral nodulus that received projections from the caudal dc and/or subnucleus $\beta$ and the rostral dc/vlo. This area is indicated with a question mark in Figures 7 and 8 . Olivocerebellar fibers from the rostral dc/vlo occupy compartment $\mathrm{XC}_{2}$ and innervate the $\mathrm{XZ}_{\text {II }}$ zone, which was also recognized by Balaban and Henry (1988) and Katayama and Nisimaru (1988). Compartment $\mathrm{XC}_{3}$ and the $\mathrm{XZ}_{\mathrm{III}}$ zone are present only in the dorsal part of the nodulus and they are innervated by the dmcc. $\mathrm{XZ}_{\mathrm{IV}}$ receives projections from the caudal dc and, in a central strip $\left(\mathrm{XZ}_{\mathrm{IV}}{ }^{*}\right)$, from the rostral dc/vlo. Our diagram differs from that of Katayama and Nisimaru (1988) because, on the dorsal surface of the nodulus, $\mathrm{XZ}_{\mathrm{TV}}$ (corresponding to their zone iv) intervenes between $\mathrm{XZ}_{\mathrm{III}}$ and $\mathrm{XZ}_{\mathrm{V}}$ (their zones $\mathrm{v}$ and vi), which are innervated by the dmce and by the rostral MAO, respectively.

Climbing fibers to the flocculus and the nodulus take their origin from different regions of the rostral MAO. Cells projecting to the flocculus of the rabbit are located in the rostral tip of the MAO (Alley et al., 1975). Projections to the nodulus of the rabbit originate from cells located along its lateral margin (Katayama and Nisimaru, 1988). The differential origin of projections to the $\mathrm{C}_{2}$ zone of the flocculus and the $X Z_{I I I}$ and $X Z_{V}$ zones of the nodulus is supported by our experiments. Injections that spared the lateral half of the rostral MAO but included the dmec (K 397 and B 2202) caused labeling in $\mathrm{XZ}_{\mathrm{III}}$, but not in $\mathrm{XZ}_{\mathrm{V}}$. Injections that included the rostral tip of the MAO (B 2202 and C 1135) labeled climbing fibers of the $\mathrm{C}_{2}$ zone.

Descriptions of the zonal arrangement of the olivocerebellar projection to the nodulus in other species are less complete and will not be discussed. The continuity of the $\mathrm{XZ}_{I}, \mathrm{XZ} Z_{T I}, \mathrm{XZ}_{I I I}$, and $\mathrm{XZ} Z_{\mathrm{Y}}$ zones with similar zones in the 
uvula is obvious from studies of this lobule in different species. $\mathrm{XZ}_{\mathrm{IV}}$ does not appear to be represented in this lobule (Brodal, 1976; Groenewegen and Voogd, 1977; Groenewegen et al., 1979; Kawamura and Hashikawa, 1979; Eisenman, 1984; Sato and Barmack, 1985, rabbit; Bernard, 1987; Kanda et al., 1989, cat; Apps, 1990, rat).

The anatomical and electrophysiological studies of Takeda and Maekawa (1984a,b 1989a,b), Maekawa et al. (1989), Kusunoki et al. (1990), and Kano et al. (1990) in the rabbit showed branching of climbing fibers between flocculus and nodulus. Takeda and Maekawa (1989) found that $35.6 \%$ of the climbing fibers in the flocculus and $63.4 \%$ of the climbing fibers in the nodulus that could be driven by optokinetic stimuli could be activated by collaterals terminating in the complementary lobule. Retrograde labeling from flocculus and nodulus with different fluorescent tracers resulted in double-labeling of 9-27\% of the dc neurons and $12-18 \%$ of the cells of the vlo (Maekawa et al., 1989). There was no distinct spatial segregation of the cells projecting to the nodulus and flocculus. Branching of olivocerebellar fibers could not be observed in our experiments, but a continuity was observed between the white matter of the flocculus and the nodulus. Fibers from the caudal dc, contained in $\mathrm{FC}_{2}$, and from the rostral dc/vlo, contained in $\mathrm{FC}_{1}$, continue from the focculus, along the attachment of the roof of the fourth ventricle into the nodulus. Furthermore, it seems likely that climbing fibers innervating the paired zones $\mathrm{FZ}_{\mathrm{I} / \mathrm{III}}$ and $\mathrm{FZ}$ II/IV of the flocculus are branches from the same axon, but this has not been definitely proved.

\section{Lateral A zone of Buisseret-Delmas (1988)}

Additional observations in our study concern the presence of a lateral A zone in the medial hemisphere of the posterior lobe (simple, ansiform, and paramedian lobules), innervated by the medial part (subnucleus c) of the caudal MAO. The olivocerebellar fibers in the white matter collect around and probably innervate the dorsolateral protuberance of the fastigial nucleus before they enter the white matter of the lobules. The lateral A zone is not continuous with its namesake (the medial A zone) in the vermis. In the simple lobule (lobule VI) the B zone intervenes between the medial and lateral A zones. A gap separating the lateral and medial A zones in lobule VII of the rat was illustrated by Akaike (1986a,b, 1987). The lateral A zone, and its climbing fiber projections from the subnucleus $c$ in the medial portion of the caudal MAO, were first distinguished by Buisseret-Delmas (1988) and Akaike (1986a,b, 1987, 1992) in the rat. The projection of group $\mathrm{c}$ of the caudal MAO to a separate zone in the hemisphere was not accounted for in the scheme of the olivocerebellar projection in the rat of Azizi and Woodward (1987), which indicated a projection of the vertical lamella of the caudal MAO, including subnucleus $c$, to a zone in the posterior vermis (their zone 4). The projection of the lateral $\mathrm{A}$ zone to the dorsolateral protuberance was substantiated by Buisseret-Delmas (1988).

\section{Course of the nucleo-olivary fibers in the rabbit}

The course of the nucleo-olivary fibers, first described by Legendre and Courville (1987) in the cat, could be confirmed because this tract was retrogradely labeled from WGA-HRP injections of the rostral dc, the vlo, and portions of the rostral MAO, DAO, and PO (but not from the caudal dc and the caudal MAO) in our experiments. This interpre- tation is supported by the absence of labeling in this pathway after injections of $\left[{ }^{3} \mathrm{H}\right]$ leucine in the inferior olive. The nucleo-olivary fibers leave the lateral and interposed nuclei passing through and around the parvicellular part of the lateral cerebellar nucleus in the rostral wall of the lateral recess. They ascend in the lateral angle of the fourth ventricle dorsal to and intermingling with the fibers of Löwy's bundle to become located ventromedial to the superior cerebellar peduncle. Subsequently they enter the decussation of the brachium conjunctivum. Nucleo-olivary fibers from the parvicellular extension of the lateral cerebellar nucleus and dorsal group y to the contralateral vlo and rostral dc (De Zeeuw et al., 1994; see also footnote 1) follow the same course.

\section{Functional considerations}

The differential projection of the dc/vlo complex to the different FZ in the flocculus is part of the neuronal circuitry responsible for the control of eye movements. With the use of microstimulation three functional zones (termed microzones by Ito, 1984) were distinguished in the rabbit flocculus either by eye movement recording (Dufossé et al., 1977; Nagao et al., 1985) or by recording the effects of vestibuloocular reflexes (VORs) on evoked eye muscle activity (Yamamoto, $1979 \mathrm{~b}$; Ito et al., 1982b). Stimulation of a narrow strip in the rostral flocculus resulted in horizontal eye movements or suppression of VORs from the horizontal canal [horizontal (H) zone II]. Stimulation rostral and caudal to zone II inhibited VORs from the anterior canal or provoked vertical eye movements [rostral and caudal, vertical (V) zones I and II]. Stimulation points of the $\mathrm{H}$ and V zones showed considerable overlap. Moreover, inhibition of the VOR for the contralateral inferior oblique muscle (Ito et al., 1982b) and rotatory eye movements could be produced by stimulation from an area in the ventral flocculus that ran obliquely through zones I-III [ rotatory (R) zone; Nagao et al., 1985]. Zone II corresponds to the more caudal of the two strips of Purkinje cells in the rabbit flocculus that project to the medial vestibular nucleus (Yamamoto and Shimoyama, 1977). Zones I and III correspond to the Purkinje cell strips projecting to the superior vestibular nucleus. [Note that the rostralmost zone of Purkinje cells projecting to the medial vestibular nucleus and described earlier by these authors was no longer mentioned in their later work (see also Ito, 1984).]

Climbing fiber responses to optokinetic stimulation were recorded as complex spike modulation of Purkinje cells of the flocculus (Kusunoki et al., 1990; see also Graf et al., 1988) and the nodulus (Kano et al., 1990) of the rabbit. Cells that preferred horizontal movement were activated when a temporally to nasally moving optokinetic stimulus was presented to the ipsilateral eye (Ipsi F cells). These cells were located in a single, $1-\mathrm{mm}$-wide strip along the caudal part of the rostral one-third of the flocculus. Some Ipsi F cells were found scattered in folium $\mathrm{p}$. There is a good correspondence between this strip and the projection of the caudal dc, which contains the vertical axis neurons of Leonard et al. (1988; see also Graf et al., 1988), to the $\mathrm{FZ}_{\mathrm{II}}$ zone. The rostromedial $\mathrm{FZ}_{\mathrm{NV}}$ zone, with a similar climbing fiber input, was not noticed by Kusunoki et al. (1990).

Most of the cells that preferred vertical optokinetic stimulation were excited by upward movement presented to the ipsilateral eye (Ipsi U cells) or downward movement presented to the contralateral eye (Contra D cells). Cells with a preference for vertical movement were located in two 
strips, one rostral and one caudal to the Ipsi F cells. These strips correspond to the $\mathrm{FZ}_{\mathrm{I}}$ and $\mathrm{FZ}_{\mathrm{III}}$ zones, which receive climbing fiber projections from the rostral dc and the vlo. The optokinetic stimulus activating the Ipsi U cells appears to be equivalent to the rotation that activated the posterior $\left(135^{\circ}\right)$ axis units in the vlo in the experiments of Leonard et al. (1988; see also Graf et al., 1988). The Contra D stimulus probably was relayed by the anterior $\left(45^{\circ}\right)$ axis units of Leonard et al. (1988; see also Graf et al., 1988) located in the rostral dc. There is a tendency for Ipsi U cells to be located more rostrally and medially in the flocculus than the Contra D cells (Kusunoki et al., 1990, their Fig. 10B,C). Similarly, the projection of the vlo to the F2 and F5 zones is medial with respect to the projection of the rostral dc to F3 and F6, as noticed by Gerrits and Voogd (1982) for the flocculus of the cat. Such a transverse gradient could not be observed in the corresponding zones in the rat (Ruigrok et al., 1992) or the rabbit.

The Purkinje cell zones in the rabbit flocculus, defined by retrograde labeling (Yamamoto and Shimoyama, 1977), microstimulation (Dufossé et al., 1977; Yamamoto, 1979b; Ito et al., 1982a,b; Nagao et al., 1985), or optokinetic stimulus-induced complex spike activity (Kusunoki et al., 1990) overlap considerably, especially in the ventral folia of the flocculus. In this respect they differ from the discrete climbing fiber projections identified in this study on the basis of the compartmental subdivision of the floccular white matter.

Purkinje cells responding with complex spikes to optokinetic stimulation have also been identified in the nodulus of the rabbit cerebellum (Simpson and Alley, 1974; Kano et al., 1990). Ipsi $F$ cells were found in medial and lateral strips in the ventral part of the nodulus. These two strips flanked a zone of Ipsi U and Contra D cells, which extended to the dorsal part of the lobule (Kano et al., 1990). This arrangement approximately corresponds to the pattern of two caudal dc-innervated zones flanking a single zone of climbing fibers from the rostral dc/vlo, as found by Katayama and Nisimaru (1988; their zones ii, iii, and iv), Balaban and Henry (1988), and by us $\left(\mathrm{XZ}_{\mathrm{I}}, \mathrm{XZ}_{\mathrm{II}}\right.$, and $\left.\mathrm{XZ}_{\mathrm{IV}}\right)$. A few Purkinje cells of the Ipsi D or Contra U type were found in the medial, subnucleus $\beta$-innervated part of zone $\mathrm{XZ}_{\mathrm{I}}$ of the nodulus and the uvula (Kano et al., 1990).

Our data indicate that the pattern of terminal zonation of the climbing fiber projection to the flocculus and nodulus is rooted in the anatomical compartmentation found with AChE histochemistry. 'This compartmentation provides an intrinsic, independent reference that is a useful tool for correlating anatomical and physiological data. This compartmentation is apparently the basis of the modular organization of differential control by the flocculus of eye muscle activity.

\section{ACKNOWLEDGMENTS}

This research was supported in part by NIH grant NS-13742. J.I. Simpson held the Tinbergen Professorship at Erasmus University Rotterdam during part of this research. The technical assistance of R.C. Boer, J. v.d. Burg. E. Dalm, E. Goedknegt, and F.II. Klink and the secretarial help of $\mathrm{E}$. Klink are gratefully acknowledged.

\section{LITERATURE CITED}

Akaike, T. (1986a) Electrophysiological analysis of the tecto-olivo-cerebellar (crus II) projection in the rat. Brain Res. 378:186-190.
Akaike, T. (1986b) Differential localization of inferior olivary neurons projecting to the tecto-olivo-recipient zones of lobule VII or crus II in the rat cerebellum. Brain Res. 386:400-404.

Akaike, T. (1987) Flectrophysiological analysis of the tecto-olivo-cerebellar (lobulus simplex) projection in the rat. Brain Res. 417:371-376.

Akaike, T. (1992) The tectorecipient zone in the inferior olivary nucleus in the rat. J. Comp. Neurol. 320:398-414.

Alley, K.A., R. Baker, and J.I. Simpson (1975) Afferents to the vestibulocerebellum and the origin of the visual climbing fibers in the rabbit. Brain Res. 98:582-589.

Apps, R. (1990) Columnar organization of the inferior olive projection to the posterior lobe of the rat cerebellum. J. Comp. Neurol. 302:236-254.

Azizi, S., and D.J. Woodward (1987) Inferior olivary nuclear complex of the rat: Morphology and comments on the principles of organization within the olivo-cerebellar system. J. Comp. Neurol. 263:467-484.

Balaban, C.D. (1984) Olivo-vestibular and cerebello-vestibular connections in albino rabbits. Neuroscience 12:129-149.

Balaban, C.D., and R.T. Henry (1988) Zonal organization of olivo-nodulus projections in albino rabbits. Neurosci. Res. 5:409-425.

Balaban, C.D., M. Ito, and E. Watanabe (1981) Demonstration of zonal projections from the cerebellar flocculus to vestibular nuclei in monkeys (Macaca fuscata). Neurosci. Lett. 27:101-105.

Barmack, N.II., M. Fagerson, and P. Errico (1993) Cholinergic projection to the dorsal cap of the inferior olive of the rat, rabbit, and monkey. J. Comp. Netrol. 328:263-281.

Bernard. J.F. (1987) 'Topographical localization of olivocerebellar and corticonuclear connections in the rat-a WGA-HRP study. I. Lobules IX, X, and the flucculus. J. Comp. Neurol. 263:241-258.

Blanks, R.H.I. (1990) Afferents to the cerebellar flocculus in cat with special reference to pathways conveying vestibular, visual (optokinetic and oculomotor signals. J. Neurocytol. 19:628-642.

Blanks, R.H.I., W. Precht, and Y. Torigoe (1983) Afferent projections to the cerebellar flocculus in the pigmented rat demonstrated by retrograde transport of horseradish peroxidase. Exp. Brain Res. 52:293-306.

Brodal, A. (1940) The cerebellum of the rabbit. A topographical atlas of the folia as revealed in transverse sections. J. Comp. Neurol. 72:63-81.

Brodal, A. (1976) The olivocerebellar projection in the cat as studied with the method of retrograde axonal transport of horseradish peroxidase. II. The projection to the uvula. J. Comp. Neurol. 166:417-426.

Brodal, A., and K. Kawamura (1980) Olivocerebellar projection: A review. Adv. Anat. Embryol. Cell Biol. 64:1-137.

Brodal, P., and A. Brodal (1981) The olivocerebellar projection in the monkey. Experimental studies with the method of retrograde tracing of horseradish peroxidase. J. Comp. Neurol. 201:375-393.

Brodal, P., and A. Brodal (1982) Further observations on the olivocerebellar projections in the monkey. Exp. Brain Res. 45:71-83.

Buisseret-Delmas, C. (1988) Sagittal organization of the olivocerebellonuclear pathway in the rat. I. Connections with the nucleus fastigii and the nucleus vestibularis. Neurosci. Res. 5:475-493.

De Zeeuw, C.I., P. Wentzel, and E. Mugnaini (1993) Fine structure of the dorsal cap of the inferior olive and its GABAergic and non-GABAergic input from the nucleus prepositus hypoglossi in rat and rabbit. J. Comp. Neurol. 327:63-82.

De Zeeuw, C.1., N.M. Gerrits, J. Voogd, C.S. Leonard, and J.I. Simpson (1994) The rustral dorsal cap and ventrolateral outgrowth of the rabbit inferior olive receive a GABAergic input from dorsal group $y$ and the ventral dentate nucleus. J. Comp. Neurol. 341:420-432.

Dufossé, M., M. Ito, and Y. Miyashita (1977) Functional localization in the rabbit's cerebellar flocculus determined in relationship with eye movements. Neurosci. Lett. 5:273-277.

Eisenman, L.M. (1984) Organization of the olivocerebellar projection to the uvula in the rat. Brain Behav. Evol. 24:1-12.

Furber, S.E., and C.R.R. Watson (1983) Organization of the olivocerebellar projection in the rat. Brain Behav. Evol. 22:132-152.

Geneser-Jensen, F.A., and T.W. Blackstad (1971) Distribution of acetylcholinesterase in the hippocampal region of the guinea-pig. I. Entorhinal area, parasubiculum and presubiculum. Z. Zellforsch. Mikrosk. Anet. 114:460-481.

Gerrits, N.M., and J. Voogd (1982) The elimbing fiber projection to the flocculus and adjacent paraflocculus in the cat. Neuroscience $7: 2971-$ 2991.

Gerrits, N.M., and J. Voogd (1986) The nucleus reticularis tegmenti pontis and the adjacent paramedian pontine reticular formation: Differential projections to the cerebellum and the caudal brainstem. Exp. Brain Res. $62: 29-45$. 
Gerrits, N.M., and J. Voogd (1989) The topographical of climbing and mossy fiber afferents in the flocculus and the ventral paraflocculus in rabbit, cat and monkey. In P. Strata (ed): The Olivocerebellar System in Motor Contral. Exp. Brain Res. Series, Vol. 17. Amsterdam: Elsevier, pp. 26-29.

Gerrits, N.M., J. Voogd, and I.N. Magras (1985) Vestibular afferents of the inferior olive and the vestibulo-cerebellar climbing fiber pathway to the flocculus in the cat. Brain Res. 332:325-336.

Gibson, A.R., D.I. Hansma, J.C. Houk, and F.R. Robinson (1984) A sensitive low artifact TMB procedure for the demonstration of WGA-HRP in the CNS. Brain Res. 298:235-241.

Giolli, R.A., R.H.I. Blanks, and Y. Torigoe (1984) Pretectal and brain stem projections of the medial terminal nucleus of the accessory optic system of the rabbit and rat as studied by anterograde and retrograde neuronal tracing methods. J. Comp. Neurol 227:228-251.

Giolli, R.A., R.H.I. Blanks, Y. Torigoe, and D.D. Williams (1985) Projections of medial accessory optic nucleus, ventral tegmental nuclei, and substantia nigra of rabbit and rat as studied by retrograde axonal transport of horseradish peroxidase. J. Comp. Neurol. 232:99-116.

Giolli, R.A., Y. Torigoe, R.H.I. Blanks, and H.M. McDonald (1988) Projections of the dorsal and lateral terminal accessory optic nuclei and of the interstitial nucleus of the superior fascicle (posterior fibers) in the rabbit and rat. J. Comp. Neurol. 277:608-620.

Graf, W., J.I. Simpson, and C.S. Leonard (1988) Spatial organization of visual messages of the rabbit's cerebellar flocculus. II. Complex and simple spike responses of Purkinje cells. J. Neurophysiol. 60:2091-2121.

Graham, R.C., and M.J. Karnovsky (1966) The early stages of absorption of injected horseradish peroxidase in the proximal tubules of the mouse kidney. Ultrastructural cytochemistry by a new technique. J. Histochem. Cytochem. 14:291-302.

Groenewegen, H.J., and J. Voogd (1977) The parasagittal zonation within the olivocerebellar projection. I. Climbing fiber distribution in the vermis of the cat cerebellum. J. Comp. Neurol. 174:417-488

Groenewegen, H.J., J. Voogd, and S.L. Freedman (1979) The parasagittal zonation within the olivocerebellar projection. II. Climbing fiber distribution in the intermediate and hemispheric parts of the cat cerebellum. $J$. Comp. Neurol. 183:551-602.

Hess, D.T., and J. Voogd (1986) Chemoarchitectonic zonation of the monkey cerebellum. Brain Res. 369:383-387.

Highstein, S.M., and H. Reisine (1979) Synaptic and functional organization of vestibulo-ocular reflex pathways. Prog. Brain Res. 50:431-442.

Hoddevik, G.H., and A. Brodal (1977) The olivo-cerebellar projections studied with the method of retrograde axonal transport of horseradish peroxidase. V. The projection to the flocculo-nodular lobe and the paraflocculus in the rabbit. J. Comp. Neurol. 176:269-280.

Holstege, G., and H. Collewijn (1982) The efferent connections of the nucleus of the optic tract and the superior colliculus in the rabbit. J. Comp. Neurol. 209:139-175.

Ito, M. (1972) Neural design of the cerebellar motor control system. Brain Res. 40:81-84.

Ito, M. (1982) Cerebellar control of the vestibulo-ocular reflex-around the flocculus hypothesis. Annu. Rev. Neurosci. 5:275-296.

Ito, M. (1984) The Cerebellum and Neuronal Control. New York: Raven

Ito, M., N. Nisimaru, and M. Yamamoto (1977) Specific patterns of neurond connections involved in the control of the rabbit's vestibulo-ocular reflexes by the cerebellar focculus. J. Physiol. (Lond.) 265:833-854.

Ito, M., Y. Miyashita, and A. Ueki (1978) Functional localization in the rabbit's inferior olive determined in connection with the vestibulo-ocular reflex. Neurosci. Lett. 8:283-287.

Ito, M., P.J. Jastreboff, and Y. Miyashita (1982a) Specific effects of unilateral lesions in the flocculus upon eye movements in albino rabbits. Exp. Brain Res. 45:233-242

Ito, M., I. Orlov, and M. Yamamoto (1982b) Topographical representation of vestibulo-ocular reflexes in rabbit cerebellar flocculus. Neuroscience 7:1644-1657

Kanda, K-I., Y. Sato, K. Ikarashi, and T. Kawasaki (1989) Zonal organization of climbing fiber projections to the uvula in the cat. J. Comp. Neurol. 279:138-148

Kano, M., M.-S. Kano, M. Kusunoki, and K. Maekawa (1990) Nature of optokinetic response and zonal organization of climbing fiber afferents in the vestibulocerebellum of the pigmented rabbit. II. The nodulus. Exp. Brain Res. 80:238-251.

Katayama, S., and W. Nisimaru (1988) Parasagittal zonal pattern of olivo-nodular projections in rabbit cerebellum. Neurosci. Res. 5:424438 .
Kaufman, G.D., J.H. Anderson, and A. Beitz (1991) Activation of a specific vestibulo-olivary pathway by centripetal acceleration in rat. Brain Res. 562:311-317.

Kawamura, K., and T. Hashikawa (1979) Olivocerebellar projections in the cat studied by means of anterograde axonal transport of labeled amino acids as tracers. Neuroscience $4: 1615-1633$.

Kooy, F.H. (1916) The inferior olive in vertebrates. Thesis. Haarlem: Bohn.

Kusunoki, M., M. Kano, M.-S. Kano, and K. Maekawa (1990) Nature of optokinetic response and zonal organization of climbing fiber afferents in the vestibulocerebellum of the pigmented rabbit. I. The floceulus. Exp. Brain Res. 80:225-237.

Langer, T., A.F. Fuchs, C.A. Scudder, and M.C. Chubb (1985) Afferents to the flocculus of the cerebellum in the Rhesus Macaque as revealed by retrograde transport of horseradish peroxidase. J. Comp. Neurol. 235:135 .

Legendre, A., and J. Courville (1987) Original trajectory of the cerebelloolivary projection: An experimental study with radioactive and fluorescent tracers in the cat. Neuroscience 21:877-891.

L.eonard, C.S., J.I. Simpson, and W. Graf (1988) Spatial organization of visual messages of the rabbit's cerebellar flocculus. I. Typology of inferior olive neurons of the dorsal cap of Kooy. J. Neurophysiol. 60:2073-2090.

Maekawa, K., and J.I. Simpson (1972) Climbing fiber activation of Purkinje cells in the flocculus by impulses transferred through the visual pathway. Brain Res. 39:245-251.

Maekawa, K., and J.I. Simpson (1973) Climbing fiber responses evoked in the vestibulocerebellum of rabbit from the visual system. J. Neurophysiol. 36:649-666.

Maekawa, K., and T. Takeda (1976) Electrophysiological identification of climbing and mossy fiber pathways from the rabbit's retina to the contralateral cerebellar flocculus. Brain Res. 109:169-174.

Muekawa, K., and T. Takeda (1977) Afferent pathways from the visual system to the cerebellar flocculus in the rabbit. In $R$. Baker and A. Berthoz (eds): Control by Gaze of Brain Stem Neurons. Amsterdam: Elsevier, pp. 187-195.

Maekawa, K., and T. Takeda (1979) Origin of descending affercnts to the rostral part of the dorsal cap of inferior olive which transfers contralateral optic activities to the flocculus. A horseradish peroxidase study. Brain Res. 172:393-405.

Maekawa, K., T. Takeda, M. Kano, and M. Kusunoki (1989) Collateralized climbing fiber projection to the flocculus and the nodulus of the rabbit. Exp. Brain Res. Ser. 17:30-45.

Martin, G.F., J. Culberson, C. Laxson, M. Linauts, M. Panneton, and I. Tschismadia (1980) Afferent connections of the inferior olivary nucleus with preliminary notes on its development: Studies using the North American opossum. In d. Courville, C.C. de Montigny, and Y. Lamarre (eds): The Inferior Olivary Nucleus: Anatomy and Physiology. New York: Raven Press, pp. 35-72.

McCrea, R.A., and R. Baker (1985) Anatomical connections of the nucleus prepositus of the cat. J. Comp. Neurol. 237:377-407.

Mizuno, N., K. Mochizuki, C. Akimoto, and R. Matsushima (1973) Pretectal projection to the inferior olive in the rabbit. Exp. Neurol. 39:498-506.

Mizuno, N., Y. Nakamura, and N. Iwahori (1974) An electronmicroscopic study of the dorsal cap of the inferior olive in the rabbit, with special reference to the pretecto-olivary fibers. Brain Res. 77:385-395.

Nayau, S. (1983) Effects of vestibulocerebellar lesions upon dynamic characteristics and adaptation of vestibulo-ocular and optokinetic responses in pigmented rabbits. Exp. Brain Res. 53:36-46.

Nagao, S. (1988) Behavior of floceular Purkinje cells correlated with adaptation of horizontal optokinetic eye movement response in pigmented rabbits. Exp. Brain Res. 73:489-497.

Nagao, S., M. Ito, and L. Karachot (1985) Eye field in the cerebellar flocculus of pigmented rabbits determined with local electrical stimulation. Neurosci. Res. 3:39-51.

Nelson, B.J., and E. Mugnaini (1989) Origins of GABAergic inputs to the inferior olive. In: The Olivocerebellar System in Motor Control. Exp. Brain Res. 17:86-107.

Nelson, B.J., N.H. Barmack, and E. Mugnaini (1986) GABAergic projection from vestibular nuclei to rat inferior olive. Soc. Neurosci. Abstr. 71:15.

Robinson, D.A. (1976) Adaptive gain control of vestibulo-ocular reflex by the cerebellum. J. Neuropłyysiol. 39:954-969.

Ruigrok, T.J.H., R.J. Osse, and J. Voogd (1992) Organization of inferior olivary projections to the flocculus and ventral paraflocculus of the rat cerebellum. J. Comp. Nourol. 316:129-150. 
Saint-Cyr, J.A., and J. Courville (1979) Projection from the vestibular nucle to the inferior olive in the cat. An autoradiographic and horseradish peroxidase study. Brain Res. 165:189-200.

Sato, Y., and T. Kawasaki (1984) Functional localization in the three zones related to eye movement control in the cat. Brain Res. 290:25-31.

Sato, Y., and N.H. Barmack (1985) Zonal organization of olivocerebellar projections to the uvula in rabbits. Brain Res. 359:281-292.

Sato, Y., and T. Kawasaki (1990a) Eye movement evoked by stimulation of Purkinje cell zones of the cerebellar flocculus in the cat. Acta Med. Biol. $38: 27-35$.

Sato, Y., and T. Kawasaki (1990b) Operational unit responsible for planespecific control of eye movement by cerebellar flocculus in cat. J. Neurophysiol. 64:551-564

Sato, Y., and T. Kawasaki (1991) Identification of the Purkinje cell/climbing fiber zone and its target neurons responsible for eye-movement control by the cerebellar flocculus. Brain Res. Rev. 16:39-64

Sato, Y., T. Kawasaki, and K. Ikarashi (1982a) Zonal organization of the floccular Purkinje cells projecting to the vestibular nucleus in cats. Brain Res. 232:1-15.

Sato, Y., T. Kawasaki, and K. Ikarashi (1982b) Zonal organization of the floccular Purkinje cells projecting to group $y$ of the vestibular complex and the lateral cerebellar nucleus in cats. Brain Res. 234:430-434.

Sato, Y., T. Kawasaki, and K. Ikarashi (1983) Afferent projections from the brainstem to the three floccular zones in cats. I. Climbing fiber projections. Brain Res. 272:27-36.

Simpson, J.I., and K.E. Alley (1974) Visual climbing fiber input to rabbit vestibulo-cerebellum: A source of direction-specific information. Brain Res 82:302-308.

Simpson, J.I., W. Graf, and C. Leonard (1981) The coordinate system of visual climbing fibers to the flocculus. In A. Fuchs and W. Becker (eds): Progress in Oculomotor Research. Developments in Neuroscience. Amsterdam: Fisevier, pp. 475-484.

Simpson, J.I., C.S. Leonard, and R.E. Soodak (1988) The accessory optic system of rabbit. II. Spatial organization of direction selectivity. J Neurophysiol. 60:2055-2072.

Takeda, T., and K. Maekawa (1976) The origin of the pretecto-olivary tract. Brain Res. 117:319-325.

Takeda, T., and K. Maekawa (1980) Bilateral visual inputs to the dorsal cap of the inferior olive: Differential localization and inhibitory interactions. Exp. Brain Res. 39:461-471.

Takeda, T., and K. Maekawa (1984a) Collateralized projection of visual climbing fibers to the flocculus and nodulus of the rabbit. Neurosei. Res. 2:125-132.
Takeda, T., and K. Maekawa (1984b) Dorsal cap neurons projecting to the flocculus, nodulus and uvula with axonal branches. Neurosci. Lett. Suppl. 17:S73.

Takeda, T., and K. Maekawa (1989a) Olivary branching projections to the flocculus, nodulus and uvula in the rabbit. I. An electrophysiological study. Fxp. Brain Res. 74:47-62.

Takeda, T., and K. Maekawa (1989b) Olivary branching projections to the flocculus, nodulus and uvula in the rabbit. II. Retrograde double labeling study with fluorescent dyes. Exp. Brain Res. 76:323-332.

Tan, J., J.I. Simpson, and J. Voogd (1995) Anatomical compartments in the white matter of the rabbit floceulus. J. Comp. Neurol. 356:1-22.

Voogd, J., and F. Bigaré (1980) Topographical of olivary and corticonuclear fibers in the cerebellum. A review. In J. Courville, C.C. de Montigny, and Y. Lamarre (eds): The Inferior Olivary Nucleus: Anatomy and Physiology. New York: Raven Press, pp. 207-234.

Voogd, J., D.'T. Hess, and E. Marani (1987) The parasagittal zonation of the cerebellar cortex in cat and monkey. In J.S. King (ed): New Concepts in Cerebellar Neurobiology, Neurology and Neurobiology. Vol. 22. New York: Alan R. Liss, Inc., pp. 183-220.

Walberg, F., N. Kotchabhakdi, and G.H. Hoddevik (1979) The olivocerebellar projections to the flocculus and paraflocculus in the cat, compared to those in the rabbit. A study using horseradish peroxidase as tracer. Brain Res. 161:389-398.

Walberg, F., T. Nordby, and E. Dietrich s (1987) The olivonodular projection A re-examination based on folial cerebellar implants. Neurosci. Lett. $81: 82-88$

Whitworth, R.H. Jr., D.E. Haines, and G.W. Patrich (1983) The inferior olive of a Prosimian primate, Galago senegalensis. II. Olivocerebellar projections to the vestibulocerebellum. J. Comp. Neurol. 219:228-240.

Yamamoto, M. (1979a) Topographical representation in rabbit cerebellar flocculus for various afferent inputs from the brainstem investigated by means of retrograde axonal transport of horseradish peroxidase. Neurosci. Lett. 12:29-34.

Yamamoto, M. (1979b) Vestibulo-ocular reflex pathways of rabbits and their representation in the cerebellar flocculus. In R. Granit and O. Pompeiano (eds): Reflex Control of Posture and Movement. Amsterdam: Elsevier/North-Holland. Progr. Brain Res. 50:451-457.

Yamamoto, M., and I. Shimoyama (1977) Differential localization of rabbit's flocculus Purkinje cells projecting to the medial and superior vestibular nuclei, investigated by means of the HRP retrograde axonal transport. Neurosci. Lett. 5:279-283. 